Revue de droit comparé du travail et de la sécurité sociale

$3 \mid 2019$

Les migrations internationales de travail

\title{
Le droit marocain dans la tourmente du nouvel ordre migratoire international
}

Rachid Filali Meknassi

\section{(2) OpenEdition}

1 Journals

Édition électronique

URL : https://journals.openedition.org/rdctss/1465

DOI : $10.4000 /$ rdctss. 1465

ISSN : 2262-9815

Éditeur

Centre de droit comparé du travail et de la sécurité sociale

Édition imprimée

Date de publication : 1 novembre 2019

Pagination : 40-71

ISSN : 2117-4350

Référence électronique

Rachid Filali Meknassi, « Le droit marocain dans la tourmente du nouvel ordre migratoire

international », Revue de droit comparé du travail et de la sécurité sociale [En ligne], 3 | 2019, mis en ligne

le 01 novembre 2021, consulté le 12 novembre 2021. URL : http://journals.openedition.org/rdctss/

1465 ; DOI : https://doi.org/10.4000/rdctss. 1465

\section{cc)}

Revue de droit comparé du travail et de la sécurité sociale est mise à disposition selon les termes de la Licence Creative Commons Attribution - Pas d'Utilisation Commerciale - Pas de Modification 4.0 International. 


\title{
LE DROIT MAROCAIN DANS LA TOURMENTE DU NOUVEL ORDRE MIGRATOIRE INTERNATIONAL
}

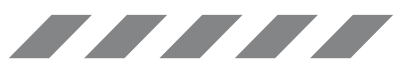

\begin{abstract}
If Morocco has long remained a country of emigration, marked by a small presence of permanent foreign residents, it seems that a new immigration and asylum politics is now in working order. The ratification of the United Nations Convention on the protection of the rights of migrant workers in 1993, the adoption of the Law 02-03 on the entry and residence of foreigners in Morocco in 2003 and the accession to the Pact of Marrakech on migration in 2018, have gradually transformed the Moroccan migration politics. Now committed to the recognition of equal rights for migrant workers and the affirmation of their fundamental rights, the country offers protective measures to immigrants, while respecting the security objectives of the European Union.
\end{abstract}

KEYWORDS: Collective Migration, Individual Migration, Migrant Law, Common Politics.

\section{RÉSUMÉ}

Le Maroc est longtemps demeuré un pays d'émigration, La conclusion des conventions bilatérales de main-d'œuvre puis de sécurité sociale, comme la ratification en 1993 de la Convention des Nations Unies sur la protection des travailleurs migrants et des membres de leur famille, ont ainsi exprimé la finalité principale de promouvoir le travail des Marocains à l'étranger et la défense de leurs droits. Mais l'arrêt de l'immigration par les pays européens depuis les années 70 et l'intégration d'une grande partie de la communauté marocaine de l'étranger dans les pays d'accueil ont considérablement réduit l'effectivité de ces normes conventionnelles. En revanche la mise en place d'une politique commune européenne dotée d'un volet sécuritaire qui associe les pays de voisinage à sa mise en œuvre a eu pour effet à la fois d'amplifier les flux migratoires sur le territoire marocain et d'imposer une nouvelle politique pour leur traitement dans le respect des droits fondamentaux des migrants et des objectifs de sécurisation des frontières convenus avec les partenaires européennes. L'adoption en 2003 de la loi relative à l'entrée des étrangers au Maroc et à la lutte contre la migration irrégulière a exprimé surtout l'adhésion à la démarche sécuritaire. L'annonce en 2013 de la nouvelle politique d'immigration et d'asile prend acte de ses limites et engage le pays dans la continuité de de la coopération avec l'Union européenne à déployer une nouvelle stratégie ouverte aussi sur les pays d'origine des migrants, dont les traits fondamentaux correspondent aux engagements du Pacte de Marrakech sur les migrations de 2018.

MOTS CLÉS: Migration collective, migration individuelle, droit des migrants, politique commune. 


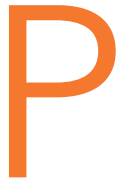

endant longtemps, la mobilité des Marocains pour le travail a été très de faible envergure et plutôt temporaire. Sous le Protectorat, une grande partie des populations rurales de l'est du pays faisait régulièrement le voyage vers les fermes d'Algérie, tandis qu'une migration ouvrière, provenant essentiellement du sud, apportait l'appoint nécessaire à un marché de l'emploi fluctuant en métropole. C'est principalement dans les conventions de main-d'œuvre conclues au lendemain de l'indépendance de l'Algérie, que la France et d'autres pays européens ont mis en place une politique d'immigration plus stable. Les accords bilatéraux conclus dans les années 60 et ceux de sécurité sociale qui les ont suivis ont aménagé des conditions favorables à la sélection des migrants, leur transfert et installation, en leur assurant l'égalité des droits au travail et à la protection sociale avec les nationaux. Les contingents de la migration collective vers la France, I'Allemagne, la Belgique et les Pays-Bas ont atteint 300000 personnes en 1974 grâce à ces mesures d'encouragement et à l'ouverture au Maroc de services de recrutement relevant des chancelleries de ces Etats.

Le retour de situation à la suite du premier choc pétrolier a mis fin à la migration collective. Mais la migration individuelle s'est poursuivie sous diverses modalités. Les restrictions apportées à la mobilité des étrangers et leur extension à la suite des Accords de Schengen, n'y ont pas mis fin. Elles auraient même eu un effet inverse en décidant ceux qui caressaient encore le projet de retour en fin de carrière à exercer pleinement leurs droits de citoyenneté, et à procéder au regroupement familial pour assurer aux membres de leur famille l'accès au territoire et la pleine jouissance des droits sociaux ${ }^{1}$. L'immigration irrégulière a elle-même donné lieu à de fréquentes régularisations en faveur des travailleurs intégrés dans l'activité professionnelle.

Cette évolution a eu pour effet de geler l'application des mesures de sélection et de transfert des émigrés. Les autres dispositions des conventions sont elles-mêmes devenues désuètes en raison de l'accès des migrants à l'égalité de traitement et aux droits de citoyenneté. Le droit marocain de l'émigration est longtemps resté conventionnel. Sa dimension institutionnelle s'est consolidée en 1990 par la création d'un Département et d'une Fondation spécialisés dans les affaires des Marocains Résidants à l'Etranger (MRE). Selon certaines estimations, leur nombre s'élève aujourd'hui à 5 millions $^{2}$, dont $45 \%$ de binationaux, confortant ainsi le sentiment que le Maroc demeure un pays d'émigration (I).

1 R. Filali Meknassi, O. Sidibé, A. Supiot, Entretien sur la politique française d'immigration, Revue Travail et Emploi: https://www.college-de-france.fr/media/alainsupiot/ UPL27 668995837309031152007 Sidibe Filali AS Entretien sur la politique franc aise d immigration.pdf

2 https://www.iom.int/fr/countries/morocco 
Ce sentiment se matérialise par la faible présence de résidents étrangers permanents sur son territoire. Sous le régime du Protectorat qui n'a duré qu'une quarantaine d'années, le peuplement européen essentiellement urbain, n'a guère dépassé les 80000 personnes. Outre les nombreux départs d'étrangers au lendemain de l'indépendance, la «marocanisation» de l'économie en 1975 a réduit davantage ses effectifs. Malgré l'ouverture économique du pays dans les années 90, on compte aujourd'hui à peine 22000 travailleurs étrangers immatriculés à la Caisse nationale de sécurité sociale. Le droit régissant leurs conditions d'emploi et de protection sociale demeure principalement constitué de dispositions du Code du travail qui ont repris les mesures instituées dans les années 30 et 40 relatives à l'autorisation préalable pour l'emploi des étrangers.

Deux faits majeurs sont venus toutefois perturber cette apparente stagnation du droit marocain des migrations, au moins au plan formel. D'une part, la ratification par le Maroc de la Convention des Nations Unies sur la protection des droits des travailleurs migrants et des membres de leur famille qui réaffirme leurs droits fondamentaux et, d'autre part, l'adoption de la loi 02-03 relative à l'entrée et au séjour des étrangers au Royaume du Maroc à l'émigration et à l'immigration irrégulières ${ }^{3}$.

Dans le contexte des restrictions européennes aux droits des migrants, le Maroc a figuré parmi les pays du sud qui ont œuvré dans l'enceinte des Nations pour la reconnaissance de l'égalité de droit en faveur des travailleurs migrants et l'affirmation de leurs droits fondamentaux, même lorsqu'ils se trouvent - en raison des nouvelles lois - en situation irrégulière. Il fut le deuxième Etat à ratifier la Convention des Nations Unies et l'un des premiers à présenter un rapport initial d'application après son entrée en vigueur en 2013. D'inspiration très différente, la loi 02-03 reproduit quasiment à l'identique les mesures de contrôle de la mobilité transfrontalière adoptées par de nombreux pays européens depuis la dévolution à l'Union Européenne de compétences en matière de migration. Elle confère aux autorités le contrôle relevant de la Direction générale de la sûreté nationale des attributions étendues, relatives à l'admission, au séjour et au travail des étrangers au Maroc. De leur exercice discrétionnaire découle la qualification de leur présence au Maroc - régulière ou non - et le recours aux mesures sécuritaires qu'elle autorise : rétention, reconduite à la frontière, expulsion, poursuite pénale, etc. Dans le silence de la loi, les droits des immigrés tendent à se définir à travers l'exercice de ce pouvoir, quand bien même la Convention des Nations Unies en indique l'étendue (II).

Cette situation paradoxale trouve naturellement son explication dans l'adhésion du Maroc à la stratégie européenne de fortification des frontières extérieures, avec l'appui des pays voisins. Alors que l'Europe vise par un tel régime répressif à éloigner les candidats à la migration de son sol, sa mise en œuvre par le Maroc a pour effet pervers de maintenir sur son territoire tous les candidats à la migration vers l'Europe. Autant les mesures de contrôle à l'entrée permettent à la police de refouler tous ceux qui lui ne la rassurent pas suffisamment sur l'objet réel de leur arrivée, autant elles s'avèrent inefficaces à l'égard de ceux qui se maintiennent au pays ou y pénètrent clandestinement au péril de leur vie, avec le dessein de traverser coûte que coûte la Méditerranée. A l'égard de ces dizaines de milliers de clandestins, embusqués aux environs des présides espagnoles de Ceuta et Melilla, guettant le départ de pateras ou cherchant à s'infiltrer dans des véhicules, les

3 Promulguée par le Dahir du 11 novembre 2003, Bulletin Officiel, n 5162 du 20 novembre 2003. Voir également son principal texte d'application, le Décret du $1^{\text {er }}$ avril 2010, Bulletin Officiel, 6 mai 2010, p. 1326. 
autorités de contrôle ne peuvent recourir qu'à la répression. Les déplacements collectifs vers les frontières de l'est et du sud, avec leur lot de violations des droits fondamentaux des personnes, se superposent aux images de naufrages répétés et de fortification des frontières, altérant le regard extérieur porté sur le Maroc et achevant de convaincre des limites d'une politique migratoire essentiellement sécuritaire.

Une nouvelle politique d'immigration et d'asile est en ordre de marche. Elle vise à concilier d'une part la poursuite des engagements sécuritaires pris à l'égard de l'Europe dans le cadre de la politique globale de mobilité et de voisinage, et d'autre part la nécessaire gestion humanitaire de la transhumance migratoire. Son succès politique s'explique davantage par son adéquation avec le consensus atone du Pacte mondial de Marrakech sur les migrations, plutôt que par son impact sur la condition des migrants et des demandeurs d'asile en situation irrégulière. Si sa mise en œuvre dépend largement de la coopération des pays de destination et d'origine, sa durabilité ne peut toutefois s'envisager qu'au travers d'un parfait équilibre entre le droit des Etats à limiter l'accès des étrangers à leur territoire, et le droit au respect de la vie et de la dignité humaines (III).

\section{I - LA CONDITION JURIDIQUE DES MIGRANTS MAROCAINS}

Les conventions bilatérales de main-d'œuvre constituent la principale source du droit marocain de l'émigration (A). Leur contenu est devenu obsolète s'agissant des mesures régissant l'émigration collective, sauf en ce qui concerne les travailleurs saisonniers (B). Ces conventions ont été suivies d'accords sur la sécurité sociale, permettant notamment la portabilité des droits $(\mathbf{C})$. Des conventions plus récentes s'appliquent à une émigration individuelle sélective (D).

\section{A - LeS CONVENTIONS BILATÉRALES DE MAIN-D'đEUVRE}

L'Allemagne fédérale a été le premier pays à conclure une convention de main-d'œuvre avec le Maroc ${ }^{4}$. L'accord signé avec la France ${ }^{5}$ quelques mois plus tard a laissé la voie ouverte à la poursuite des flux migratoires telle que confiée à l'Office national d'immigration depuis 1945. Son contenu a inspiré les accords qui furent conclus par la suite avec la Belgique en $1964^{6}$ et les Pays-Bas en 1969. Les conventions ultérieures avec l'Espagne (2001) et l'Italie (2005) reproduisent des mesures similaires, sans faire état de la sélection et de l'envoi de travailleurs.

4 La RFA avait auparavant conclu des accords de main-d'œuvre industrielle avec l'Italie (1955), l'Espagne (1960) et la Turquie (1961) selon un modèle de rotation empêchant l'installation durable. Ce n'est qu'en 1970 que les étrangers ont été autorisés à être rejoints par les épouses et les enfants, d'où la faible présence de la communauté marocaine en Allemagne. La Convention avec le Maroc a été conclue le 21 mai 1963.

5 Convention de main-d'œuvre entre la France et le Maroc du 1 1er juin 1963, entrée en vigueur en France par le Décret $n^{\circ}$ 63/779 du 27 juillet 1963 portant publication de la Convention de maind'œuvre entre la France et le Maroc du 1 er juin 1963.

6 La Convention conclue avec la Belgique n'a été publiée que le 17 juin 1977, en même temps que d'autres conventions bilatérales, sans doute pour éviter la revendication de l'alignement de son contenu avec les accords plus favorables conclus avec d'autres pays, notamment la Turquie. 
Renouvelables par tacite reconduction, ces accords engagent les parties à simplifier les formalités administratives pour le départ du Maroc, l'entrée dans le pays d'accueil, la délivrance des titres de séjour et de travail, et la réduction des frais y afférents. Ils permettent de recourir aussi bien au recrutement nominatif qu'anonyme. Dans tous les cas, l'exercice de l'activité professionnelle demeure suspendu à la conclusion d'un contrat de travail visé par l'autorité chargée des questions de travail au sein du pays d'emploi. Ils prescrivent, parfois en annexe, les modalités de mise en œuvre relatives à la sélection des candidats, la prise en charge des frais de voyage et la formation.

Le principe de base consiste en une égalité de traitement entre les travailleurs migrants et les travailleurs nationaux du pays d'emploi en ce qui concerne les conditions de travail, de rémunération, et de logement. Le droit aux allocations de chômage et au transfert des revenus est garanti. II est également prévu qu'en cas de non engagement ou de rupture du contrat de travail, des mesures soient prises par les services administratifs compétents pour l'insertion du travailleur dans des conditions similaires. Le regroupement familial est autorisé. La Convention conclue avec la France l'énonce en ces termes: «les familles des travailleurs marocains peuvent rejoindre ces derniers et toutes facilités seront accordées à cet effet par le Gouvernement français dans le cadre de la législation et de la réglementation actuellement en vigueur ${ }^{7}$. Des recommandations sont adressées aux employeurs pour permettre aux travailleurs migrants de jouir des jours fériés et chômés au Maroc. Par ailleurs, une commission mixte est instituée, afin de suivre l'exécution de la Convention, de proposer le cas échéant sa révision et d'aplanir toute difficulté d'application.

\section{B - LE DROIT APPLICABLE AUX MIGRATIONS TEMPORAIRES}

La migration saisonnière agricole des Marocains n'est pas nouvelle. Très développée sous le Protectorat entre l'est du pays et les exploitations agricoles d'Algérie, elle a aussi connu un certain essor vers la France méridionale grâce aux contrats dits $\mathrm{OMI}$, dénomination qui subsiste malgré les transformations de cet établissement public ${ }^{8}$. L'élargissement de I'Union Européenne a occulté sa visibilité dans la mesure où les travailleurs espagnols, portugais et yougoslaves qui alimentaient les statistiques migratoires ont cessé d'être

7 Voir art. 11 de la Convention franco-marocaine. La Convention signée avec la Belgique l'année suivante a été suivie de l'adoption, en 1965, d'une réglementation qui prévoit que la moitié des frais de voyage de l'épouse et des enfants qui l'accompagnent seront remboursés si la famille compte au moins trois enfants mineurs. L'encouragement en faveur du regroupement familial a fait l'objet d'une brochure du ministère de l'emploi et du travail intitulée: "Vivre et travailler en Belgique ». Pour attirer les mineurs, son annexe prévoit aussi de mettre à la disposition des travailleurs un «logement convenable et une nourriture conforme (...) à leurs habitudes alimentaires ».

8 L'Office des Migrations Internationales (OMI) est l'appellation donnée en 1988 à l'Office national d'immigration (ONI). Celui-ci a reçu en 1945 le monopole du recrutement, de l'acheminement et du placement des travailleurs étrangers en France. Financé par les « redevances forfaitaires » versées par les employeurs lors de la signature de chaque contrat de travail, il a été rebaptisé en 2005 Agence nationale de l'accueil des étrangers et des migrations (ANAEM), puis Office français de l'immigration et de l'intégration (OFI) en 2009. Désormais sous tutelle du Ministère de la migration et non plus du travail, il a perdu son monopole sur le recrutement des migrants. Voir A. Morice, "Quelques repères sur les contrats OMI et ANAEM », in Travailleurs saisonniers dans l'agriculture européenne, Etudes Rurales, n 182, 2008; Décret n²009-331 du 25 mars 2009: http://www.ofii. fr/qui-sommes-nous? 
considérés étrangers. Au final, 10000 à 15000 saisonniers maghrébins font le voyage pour assurer les récoltes dans les serres et les vergers du sud de la France.

Pour le Maroc, comme pour la Tunisie, la durée du séjour ne doit pas être inférieure à 4 mois ni supérieure à 6 mois, sauf dérogation autorisant soit un temps plus court si l'employeur s'engage à prendre en charge le retour du travailleur, soit 2 mois supplémentaires?.

Les contrats de l'Agence nationale de l'accueil des étrangers et des migrations (ANAEM ci-après) donnent lieu à la délivrance d'une carte de séjour temporaire (CST) pour une durée maximale de trois ans renouvelable. Le droit au séjour en France est limité à la période fixée et ne saurait excéder six mois par an, le travailleur s'engageant à maintenir sa résidence hors de $\mathrm{France}^{10}$. A son retour, il est tenu de se présenter à la délégation de I'OFI à Casablanca. Depuis cette année, celle-ci a cessé de prendre en charge les coûts du voyage. L'accord hispano-marocain s'en inspire tout en profitant des mesures de soutien instituées par I'Union Européenne. II n'a été rendu public que 6 années après sa conclusion ${ }^{11}$. Toutefois, l'article $19 \S 2$ prévoit son application provisoire 30 jours à compter de la date de signature ${ }^{12}$. En conséquence, il a pu dès 2004 servir de support à la conception et à la mise en œuvre du Schéma de migration saisonnière instauré avec la municipalité de Huelva. Comparativement aux conventions de main-d'œuvre conclues antérieurement, la nouveauté de cet accord réside dans l'inclusion d'un titre réservé à la migration saisonnière, sans pour autant aborder réellement ses spécificités. C'est essentiellement par des arrangements entre les administrations concernées que les conditions de sa mise en œuvre se déterminent.

La Convention use de la nouvelle terminologie afférente à la régulation « de façon ordonnée et coordonnée des flux de la main-d'œuvre entre les deux pays ». Son champ d'application concerne les seuls « citoyens marocains autorisés à exercer pour le compte d'autrui une activité rémunérée sur le territoire espagnol ». L'Ambassade d'Espagne à Rabat est chargée de communiquer aux autorités marocaines les besoins en main-d'œuvre tels qu'ils lui parviennent des employeurs espagnols ou de leurs représentants. Par le même canal, les autorités marocaines lui adressent, en retour, les informations sur les possibilités de les satisfaire.

9 A. Morice et B. Michalon, "Les migrants dans l'agriculture: vers une crise de main-d'œuvre?», Etudes Rurales, $\mathrm{n}^{\circ} 182,2008$, p. 9.

10 En 2016, le Maroc occupait la 2e place après l'Albanie avec $15 \%$ du total des migrants saisonniers arrivés dans les territoires de l'UE.

11 La signature de cet Accord a suivi l'adoption d'une loi sur la migration en 2000 qui a permis de procéder à une large régularisation. Mais suite à un changement de majorité, une politique de restrictions à la migration a été mise en place, donnant la préférence à la conclusion d'accords de main-d'œuvre avec le Maroc (2001). Voir: https://www.fidh.org/IMG/pdf/rapport fraises fr.pdf

12 Publié en vertu du Dahir du 17 avril 2007 B.O. n 5532 du 7 juin 2007, I'accord a été conclu le 25 juillet 2001 par le Premier ministre marocain et le Chef du gouvernement espagnol. Son article 2 désigne comme autorité compétente du côté marocain le Ministère du travail, et du côté espagnol les Ministères des affaires étrangères, de l'intérieur et du travail dans le cadre de leur compétence respective en matière d'immigration. 
Les offres peuvent être nominatives ou anonymes. La fidélisation des recrues par les employeurs espagnols s'effectue par le renouvellement de leur engagement et l'octroi de permis de séjour et de travail, sans passer par la procédure collective ${ }^{13}$.

Une Commission paritaire de sélection est prévue. Elle doit «sélectionner les travailleurs dont le profil professionnel répond aux offres d'emploi existantes, organiser les cours de formation préalable nécessaires, conseiller et assister les travailleurs tout au long du processus de recrutement. Pourraient également faire partie de cette Commission l'employeur ou son représentant ».

Les travailleurs retenus après examen médical signent le contrat de travail et reçoivent leurs billets de voyage-aller, le retour restant à leur charge. Le visa de séjour ou de résidence leur est délivré par les services consulaires dans les plus brefs délais et le cachet qui en atteste sur le passeport indique la finalité et la durée du séjour autorisé. II tient lieu de permis de travail, chaque fois que cette durée est inférieure à 6 mois.

Les autorités marocaines facilitent les opérations de sélection, de formation et de départ. Outre une copie de leur contrat, les personnes retenues reçoivent un document écrit indiquant les informations relatives à leur séjour, emploi, logement et rémunération. Les autorités espagnoles fournissent quant à elles les permis correspondant à leur travail et séjour.

L'Accord prévoit l'égal exercice des droits au travail conférés par la législation espagnole et les conventions collectives. Le transfert des revenus s'effectue dans le respect de la législation de change. La convention bilatérale sur la sécurité sociale est appliquée.

Les modalités conjointes d'application de l'Accord relèvent du côté marocain du Ministère du travail par le biais de la direction de l'emploi, et du côté espagnol du Ministère de l'intérieur par le biais de la délégation du Gouvernement pour les étrangers et l'immigration. L'Accord fait référence à « des engagements déjà souscrits par les deux pays pour renforcer leur coopération dans le domaine du contrôle de la législation du travail, notamment afin d'éviter l'exploitation des Marocains en situation irrégulière ${ }^{14}$.

Un comité mixte de coordination est constitué pour suivre l'exécution de l'Accord, proposer son amélioration, diffuser l'information, résoudre les difficultés d'application, etc.

La définition du travailleur saisonnier exclut l'application de l'Accord aux Marocains régulièrement installés en Espagne. Elle confère une flexibilité extrême à la durée du séjour convenu et au temps du travail effectif correspondant, tout en insérant les opérations y afférentes au dispositif commun applicable à la migration de la main-d'œuvre marocaine, en ce qui concerne la sélection, le déplacement et l'accueil en Espagne. L'art 14 déclare toutefois que « les conditions de séjour et de retour des travailleurs saisonniers feront l'objet

13 Les travailleuses ainsi fidélisées sont qualifiées de "répétitrices» (repeticiones) en Espagne. L'article 13 déclare que "les demandes d'autorisation de séjour et de travail annuelles et renouvelables, formulées par les travailleurs marocains ayant exercé en Espagne pendant 4 ans consécutifs ou non, à titre de saisonniers, seront examinées par la partie espagnole, avec une bienveillance particulière ».

14 Le rapport de la FIDH précité fait état d'un accord administratif relatif aux travailleurs saisonniers marocains en Espagne signé à Madrid le 30 septembre 1999 pour une durée de 3 ans renouvelable par tacite reconduction (p. 21, note 28 ). 
d'un engagement écrit des intéressés vis-à-vis des autorités espagnoles, conformément aux lois et règlements en vigueur dans le pays d'accueil $»^{15}$.

La province de Huelva, qui assure $90 \%$ des exportations de fraises, est le principal artisan de cette expérience ${ }^{16}$ qui a bénéficié du soutien décisif de I'UE ${ }^{17}$. Ce dispositif cible en particulier les travailleuses agricoles âgées de 25 ans à 45 ans, mères de 2 enfants au moins ${ }^{18}$, moins disposées à changer d'employeur ou à s'installer définitivement dans le pays. Le nombre de Marocaines parmi les migrantes saisonnières à Huelva est ainsi passé de 200 en 2001, à 14000 en 2009. Après avoir chuté au cours de la crise économique, il a rebondit au cours des deux dernières années, pour atteindre 16000 en 2019.

Le modèle de contrat usuel est exclusivement rédigé en espagnol. C'est un contrat type que la travailleuse signe avant d'être engagée ${ }^{19}$. Conclu en principe pour une durée de 3 mois en ce qui concerne la Province de Huelva, le séjour peut toutefois être écourté en cas de surplus de main-d'œuvre ou, au contraire, rallongé jusqu'à 9 mois comme l'autorise la législation du travail. La durée hebdomadaire de travail est limitée à 39 heures, conformément à la législation en vigueur. Ce plafond reste cependant indicatif. Le contremaître désigne d'autorité les tâches et leur durée. Son pouvoir discrétionnaire détermine ainsi les revenus du travail et l'identification des "repeticiones» qui seront nommées dans l'établissement des besoins de la saison suivante ${ }^{20}$.

15 L'engagement que les migrantes signent pour le travail à Huelva leur impose de se présenter personnellement au Consulat d'Espagne dans le mois qui suit leur retour.

16 Voir D. Zeneidi, «Femmes/fraises Import/export ch1, Global food et migration utilitaire dans la province de Huelva ", PUF, 2013, p. 9; voir également https://www.cairn.info/femmes-fraises-9782130624585-page-27.htm

17 La ville a obtenu en 2004 un financement européen de 1196000 euros dans le cadre du programme Aeneas (Programme of assistance to third countries directed related to migration management) pour mettre en place un outil d'encadrement des ouvrières marocaines. Ce programme a été prolongé jusqu'en 2013 par un nouveau financement attribué par l'Union Européenne. Voir: https://halshs.archives-ouvertes.fr/halshs-01525363 (p. 4, note 6).

18 Selon le rapport précité de la FIDH (p. 29), l'année 2002 a connu un taux de fuite des femmes saisonnières qui dépassait les $90 \%$ sur un total de 500 . Il est resté élevé jusqu'à la mise en place de procédures de recrutement par les gouvernements espagnol et marocain et seront affinées dans le cadre du projet AENEAS Cartaya. Le directeur de I'ANAPEC assure que le taux de retour s'établit depuis 2005 à au moins $95 \%$.

19 Un accord national a été signé en 2006 entre les autorités centrales, les syndicats et les organisations patronales agricoles portant sur l'organisation des flux migratoires dans les campagnes agricoles saisonnières. Sa mise en œuvre est confiée à une Commission au niveau étatique, ainsi qu'à des commissions provinciales en charge de l'analyse, de la planification des besoins en main-d'œuvre, de l'établissement du nombre de travailleurs nécessaires, de la localisation de la main-d'œuvre, de l'organisation de la venue des travailleurs et de leur intégration.

20 L'examen du contrat annexé au rapport de la FIDH confirme qu'il prévoit 3 mois au minimum de travail et une période étalée de janvier à juin (art 3). L'art 4 prescrit une durée de travail hebdomadaire de 39 heures de lundi à dimanche, ce qui semble exclure le repos hebdomadaire (art 5). L'art 7 renvoie à la convention collective en ce qui concerne les journées de chômage technique. Or cette dernière prévoit l'indemnisation des seuls travailleurs ayant travaillé pendant 270 jours, ce qui exclut les saisonniers. En revanche, le modèle de contrat examiné auprès des travailleuses fait état de 3 mois fermes. II semble que c'est à ces caporaux qu'ont été imputées les allégations de harcèlement sexuel. 
Le salaire varie entre 34 et 37 euros par jour. L'employeur assure le logement ainsi que les frais de transport de la ville de Tanger vers le lieu de travail. Les ouvrières supportent les coûts de visa espagnol, les frais du passeport, et les dépenses de trajet de leur domicile au point d'embarquement à Tanger.

Au Maroc, c'est l'agence nationale ANAPEC qui a été désignée pour gérer ces opérations, avant même sa création. Le montage institutionnel a également bénéficié de l'appui technique et financier du programme européen AENEAS dans le cadre de l'Accord de MEDA II. II prévoit la création au sein de la future ANAPEC d'une Division Internationale (DI) et de 4 agences régionales. L'expérience figure aujourd'hui sur le site du partenariat entre l'UE et l'Union Africaine comme relevant d'une «bonne pratique $»^{21}$.

La Convention signée en 2010 entre la FUTEH ${ }^{22}$ et l'ANAPEC prévoit la garantie par les employeurs contractants de:

- 18 jours de travail effectif au minimum par mois;

- la déclaration à la sécurité sociale;

- la non rétention des passeports pendant le séjour;

- la prise charge du coût total du voyage de retour par l'entreprise en cas de licenciement du travailleur dans les 15 premiers jours.

La partie espagnole se charge de l'accueil, du transfert et des retours. Le suivi est assuré par des médiateurs relevant de la mairie de Cartaya. Les inspecteurs de travail et les syndicats supervisent le respect des conditions légales du côté espagnol. L'ANAPEC est admise sur les lieux ${ }^{23}$.

Malgré la pénibilité du travail et les aléas de cette migration, elle est fortement sollicitée. Les conditions de travail et de rémunération qu'elle garantit sont nettement meilleures et plus sûres que celles qui prévalent au Maroc dans la même activité, notamment dans les exploitations espagnoles.

21 Voir : https://www.africa-eu-partnership.org/fr/success-stories/appui-institutionnel-la-circulationde-personnes-aux-fins-de-travail-entre-lue-et-le. Le projet a aussi été cofinancé par le Maroc et a bénéficié de l'assistance technique d'un consortium composé d'établissements publics français (Pôle Emploi, Office Français de l'Immigration et de l'Intégration), espagnols (FIIAPP - Fondation Internationale pour l'Amérique Latine d'Administration et Politiques Publiques) et belges (FOREM - Office Wallon de la Formation Professionnelle et de l'Emploi et ACTIRIS -Office Régional Bruxellois de l'Emploi).

22 Fondation pour les travailleurs étrangers à Huelva, créée par le patronat à Cartaya.

23 Un communiqué commun rapporte la signature par le Ministre du travail et de l'insertion professionnelle, et le Ministre délégué chargé des Marocains résidant à l'étranger et des affaires de la migration, d'une Convention "pour améliorer la procédure d'emploi des travailleuses saisonnières dans les exploitations agricoles espagnoles, leur garantir des conditions de travail décent et établir un mécanisme de suivi tout au long de la saison agricole ». Elle prévoit également la mise en place d'une commission mixte pour effectuer des visites dans les exploitations agricoles, afin de suivre les conditions de travail des travailleuses saisonnières, en coordination avec les autorités espagnoles compétentes. 


\section{C - LES CONVENTIONS BILATÉRALES DE SÉCURITÉ SOCIALE}

L'égalité de traitement entre les migrants et les ressortissants du pays d'accueil en matière de sécurité sociale a comme corolaire la conservation des droits à la sécurité sociale. Sur les 18 conventions conclues par le Maroc à cet effet, 15 sont en vigueur. La portabilité des droits qu'elles devraient garantir éprouve néanmoins de nombreuses difficultés de mise en œuvre.

Les accords bilatéraux de sécurité sociale ont pour objet commun de:

- supprimer les dispositions discriminatoires fondées sur la nationalité;

- adapter les restrictions affectant le champ d'application territoriale de la législation;

- soutenir l'élaboration de carrières d'assurance pour les migrants;

- instaurer une coopération entre les institutions et les organismes nationaux de sécurité sociale.

Les conventions reposent généralement sur le principe de la territorialité de la sécurité sociale. Il est courant toutefois d'écarter le principe d'application de plein droit de la règle d'égalité pour certaines catégories de travailleurs, à l'instar des salariés détachés et des travailleurs au sein d'entreprises de transport.

Lorsque le travailleur et les membres de sa famille résident dans le pays d'emploi, leur couverture est garantie, mais des différences apparaissent d'une part, lorsque le travailleur ou les ayants droits se trouvent provisoirement hors du pays d'emploi, d'autre part lorsque la famille du travailleur est restée dans le pays d'origine.

Ainsi la Convention avec la Suède retient à ce sujet la règle d'égalité de traitement (art. 3) et celle de cumul des périodes d'assurance dans les deux pays (art. 10), sans décider expressément de la solution à appliquer eu égard aux délais minimums à respecter pour prétendre à indemnisation. En revanche, l'article 5 de la Convention avec l'Allemagne écarte toute règle pouvant suspendre le bénéfice des prestations en nature ou en espèces à une condition de stage. De leur côté, les Conventions passées avec la France et l'Espagne disposent simplement que seules les règles du pays de résidence reçoivent application. Elles prévoient explicitement la couverture des membres de la famille restés au pays d'origine, alors que les Conventions avec le Danemark et la Suède n'abordent pas la question. Pour sa part, la Convention avec l'Allemagne renvoie à l'adoption d'un texte d'application qui tiendrait compte de l'état du droit dans les deux pays.

La question du séjour à l'étranger de l'assuré et de ses ayants-droits connaît aussi des différences dans le traitement formel. Dans la Convention avec la France, seul le congé annuel dans le pays est mentionné. En revanche, la Convention avec l'Espagne ajoute à celui-ci les absences autorisées. D'autres conventions enfin mentionnent la résidence temporaire (Belgique, Allemagne, Portugal et Pays-Bas) ou plus généreusement le séjour à l'étranger (Danemark).

En ce qui concerne le transfert de résidence durant le congé de maladie, certaines conventions disposent que pour avoir droit à lıindemnisation, il est nécessaire qu'il ait été préalablement autorisé ou qu'il donne lieu à régularisation. En cas de retour définitif au pays, les nouvelles conventions passées avec la France et l'Espagne prescrivent explicitement le maintien des prestations, alors que d'autres conventions n'abordent pas l'hypothèse.

S'agissant de la réparation des accidents de travail et des maladies professionnelles, le maintien de la couverture en cas de changement de résidence est prévu par toutes les 
conventions. Les accords passés avec le Portugal, la Belgique et le Danemark, spécifient néanmoins qu'il doit s'agir de séjour au pays d'origine. Le versement des allocations familiales est prévu, abstraction faite du lieu de résidence. Leur montant peut être différent selon que les enfants se trouvent dans le pays d'emploi ou non (France, Belgique, Allemagne). La Convention avec la Suède est muette à ce sujet, et celle du Danemark prévoit des arrangements administratifs au sujet des enfants qui restent dans le pays pour des nécessités liées à leur éducation. Toutes les conventions retiennent le maintien et le transfert des droits à pension d'invalidité, de retraite et d'ayants-droit.

Pour la France et la Belgique, les modifications récentes introduites dans les conventions bilatérales de sécurité sociale concernent l'extension des prestations de maladies aux assurés installés définitivement au Maroc. En revanche, les Pays-Bas ont décidé de réduire progressivement le montant des allocations familiales servies au titre des enfants installés au Maroc, à concurrence de 40 \% en janvier 2019, et leur arrêt définitif en janvier 2021.

La portabilité des droits sociaux présente un intérêt particulier pour la décision de retour des migrants au pays et celle de regroupement familial. Une récente étude sur la question a ciblé les droits à la retraite des migrants marocains ${ }^{24}$. Ce Rapport (Projet Sharaka) rapporte qu'en 2010-2011, plus de 266000 Marocain(e)s âgé(e)s de plus de 65 ans, majoritairement de sexe féminin résidaient dans les pays de l'OCDE. Deux tiers d'entre eux étaient sur le marché de travail, mais leur taux d'emploi était de 47 \% seulement et celui du chômage de $29 \%$. La majorité des retraités se trouvaient en situation de précarité, subsistant par des pensions partielles et les minima sociaux. La tendance est appelée à s'aggraver. La majorité de ces migrants ne parvient pas à concrétiser le vœu de retour au pays, faute de sécurité économique et juridique. Outre les raisons familiales, notamment le souhait de maintenir une proximité avec les enfants et petits-enfants, leur transhumance est motivée par des logiques plus économiques liées à la pérennisation des droits sociaux acquis, ou le bénéfice d'autres aides non ouvertes à la portabilité (versement des pensions, accès aux soins de santé, minimums vieillesses complémentaires, aides au logement, etc.).

En outre, les emplois précaires qui se sont multipliés avec la crise économique et qui ont davantage touché les travailleurs immigrés, risquent de peser au moment de la retraite. Aussi, les conditions semblent réunies pour que progresse, dans les années à venir, le nombre de personnes en difficulté chez les migrants âgés, particulièrement ceux qui ont séjourné en Espagne et en Italie.

Le Rapport estime que la problématique de la portabilité des droits des retraités concerne prioritairement les personnes qui sont rentrées définitivement au Maroc, ainsi que leurs ayants droit bénéficiant des droits dérivés. La diversité des régimes de pensions et leur articulation avec les autres prestions sociales compliquent le schéma. L'ouverture de ces droits repose sur des conditions marquées par des différences importantes entre pays, tant aux niveaux des durées de cotisation, d'âge, et d'ouverture des droits, qu'au niveau des pensions.

24 Projet Sharaka, la portabilité des droits sociaux: http://marocainsdumonde.gov.ma/wp-content/ uploads/ 2019/01/\%C3\%89tude-sur-la-portabilit\%C3\%A9-des-droits-des-retrait\%C3\%A9s-MREau-Maroc.pdf 
La migration saisonnière constitue sans doute aussi un risque de contribution des migrants à un régime de sécurité sociale étranger, sans perspective réelle d'accéder aux prestations sociales qu'il institue au terme de périodes de stages, difficilement atteignables par eux.

\section{D - L'AMÉNAGEMENT D'UNE MIGRATION EN COLS BLANCS}

Loin des accords bilatéraux de main-d'œuvre, d'autres conventions sont conclues pour organiser la mobilité transfrontalière par l'octroi des visas, le rapatriement et le séjour régulier. ${ }^{25}$ Elles s'adressent à des cibles différentes.

La plupart des étudiants post-masters éprouvent peu de difficulté à s'engager, dès la fin de leurs études, dans une activité professionnelle en Europe, au Canada et aux USA. Les conditions matérielles, les perspectives de carrière, l'environnement socioculturel et la prise en compte de leur séjour antérieur pour l'obtention de la nationalité concourent à favoriser cette option, même si un retour ultérieur au pays n'est pas écarté. De nombreux Marocains deviennent binationaux, avant même l'échéance de leur première ou seconde carte de séjour. Ils sont rejoints par plusieurs lauréats de grandes écoles marocaines, déçus par les faibles possibilités d'emploi et de carrière que leur offre le marché de travail local, ainsi que par des profils professionnels supérieurs saisissant les opportunités de reconnaissance et de promotion que leur offrent les maisons-mères et les réseaux dans lesquels les insère leur activité. II semble même que la migration des jeunes cadres soit devenue un signe de réussite intellectuelle et/ou professionnelle. L'expression "fuite de cerveaux » restitue imparfaitement ses motivations et son ampleur.

L'immigration choisie s'inscrit dans la compétition scientifique et technologique, comme l'a affirmé la stratégie européenne de Lisbonne. La plupart des législations prévoient l'octroi de titres de séjour et de travail à tous les cadres et titulaires de diplômes supérieurs pour la durée de leur premier CDD et pour une durée d'un an au moins renouvelable de plein droit en cas de poursuite de l'engagement par CDI. Le premier titre de séjour et de travail est remplacé par une carte de séjour ou de résidence dès que l'attributaire justifie de la continuité de l'exercice d'une activité professionnelle lui procurant des revenus suffisants. C'est sous cet angle que doit être appréhendé l'Accord en matière de séjour et d'emploi, conclu à Rabat le 9 octobre 1987 entre le Royaume du Maroc et la République française ${ }^{26}$.

A la différence des conventions de main-d'œuvre qui régissent l'émigration collective, cette Convention aborde dans le cadre de la réciprocité, la délivrance des titres de séjour et de travail aux ressortissants de chaque pays par l'autre partie.

25 Voir notamment l'Accord conclu à Madrid le 13 février 1992 entre le Maroc et l'Espagne relatif à la circulation des personnes, au transit et à la réadmission des étrangers entrés irrégulièrement, qui a fait l'objet de publication par le Dahir du 2 août 2012, (BO nº 6214 du 19 décembre 2013).

26 Publié par le Dahir du 2 août 2011, B.O. nº 6080 du 6 septembre 2012. 
Ses dispositions décident que les Marocains, titulaires à la date de son entrée en vigueur d'un titre de séjour d'une durée de 3 ans au moins, reçoivent de plein droit à son terme une carte de séjour valable pour 10 ans au moins. Cette carte est renouvelable de plein droit et vaut « autorisation de séjourner sur le territoire de la République française et d'exercer, dans ses départements européens, toute profession salariée ou non ${ }^{27}$.

Les Marocains ne relevant pas de cette catégorie, désirant exercer une activité salariée en France pour une durée d'une année au minimum, reçoivent sur présentation d'un contrat de travail visé par les autorités compétentes un titre de séjour renouvelable, valable une année et portant la mention « salarié », avec éventuellement des restrictions géographiques ou professionnelles. Après 3 ans de séjour continu en France, ils peuvent obtenir le titre de séjour de 10 ans. Il est statué sur leur demande en tenant compte des conditions d'exercice de leurs activités professionnelles et de leurs moyens d'existence. Des règles identiques s'appliquent aux ressortissants français remplissant les mêmes conditions au Maroc.

Les conjoints et les enfants mineurs bénéficiaires de regroupement familial sur le territoire de l'un et l'autre Etat sont admis, quelle que soit la date de leur arrivée, à y résider dans les mêmes conditions que les titulaires des autorisations de séjour et de travail. Ils accèdent dans les mêmes conditions à l'emploi, sur présentation d'un contrat visé par l'autorité compétente, sans que la situation de l'emploi puisse leur être opposée (art. 7).

Sous réserve des mesures communes européennes, les durées plus longues accordées aux titres de séjour et de travail par l'un des Etats, aux ressortissants de l'autre, entrainent réciprocité.

Il est vrai que la stratégie de Lisbonne avait fixé comme objectifs à l'Union de développer une politique commune des migrations visant, d'une part l'attraction des meilleurs profils pour gagner la bataille de la compétitivité aux plans technologique et scientifique, et d'autre part la lutte contre la migration irrégulière. Le premier n'a pas besoin de la coopération bilatérale pour se réaliser, tandis que le second est tributaire de l'intégration de la coopération sécuritaire dans la vision euro-méditerranéenne par une politique dite « de voisinage ».

\section{II - LA CONDITION JURIDIQUE DES IMMIGRÉS AU MAROC}

L'inclusion du pays dans la stratégie européenne de la migration a conduit le Maroc à se doter d'une législation de lutte contre la migration et l'immigration irrégulières (A). Son application dévoile les limites du dispositif légal applicable au travail des étrangers et tend à lui conférer une position faitière (B).

27 Une mesure similaire est prévue pour les ressortissants français régulièrement installés sur le territoire marocain depuis 3 ans. Il leur est délivré de plein droit, pour 10 ans, une autorisation de séjour et visa par les autorités compétentes les autorisant à exercer toute profession salariée, sur toute l'étendue du territoire. A son échéance décennale, l'autorisation de séjour et de visa de travail sont renouvelés de plein droit pour 10 ans. 


\section{A - UN DROIT DE L'IMMIGRATION DOMINÉ PAR LES DISPOSITIFS DE LUTTE CONTRE LES MIGRATIONS IRRÉGULIÈRES}

Inspirée par la nouvelle législation française et rédigée dans le contexte de la négociation de l'Accord de coopération MEDA avec I'Union Européenne, la Loi 02/03 a été adoptée par le Parlement, promulguée par le Roi et publiée au Bulletin Officiel avec une rapidité extrême pour prendre effet le 20 novembre $2003^{28}$. Un texte similaire a été adopté par l'Espagne à une semaine d'intervalle ${ }^{29}$. Ses dispositions posent les règles de base d'admission et de séjour des étrangers au Maroc. Leur mise en œuvre et la sanction de leur violation sont confiées aux autorités de contrôle placées sous l'autorité du Directeur général de la Sûreté Nationale.

La nouvelle loi abroge et remplace les dispositions antérieures applicables, tant à l'entrée et au séjour des étrangers au Maroc qu'à la soumission des migrants à l'autorisation administrative préalable pour exercer une activité professionnelle ${ }^{30}$. Elle confère aux autorités de de larges attributions pour décider de l'admission des étrangers sur le territoire à leur arrivée, de l'octroi de titres de séjour, de leur retrait et annulation, ainsi que de leur maintien dans des zones de rétention, leur reconduite à la frontière et leur expulsion.

La règle de base présume que l'entrée sur le territoire s'effectue par les postes de contrôle frontalier et doit être attestée par le cachet porté sur le document de voyage. Toute personne qui n'est pas en mesure d'en justifier l'existence se trouve en situation irrégulière. Le contrôle à l'entrée porte sur les documents de voyage, les moyens d'existence, l'objet du séjour et les garanties de rapatriement. L'admission est refusée lorsque les agents commis à cette fonction estiment que les justificatifs présentés sont insuffisants, ou que la présence de l'étranger constituerait « une menace pour l'ordre public ». L'étranger est alors renvoyé par le même moyen de transport qu'il a emprunté pour arriver. II a le droit d'aviser la personne à laquelle il a déclaré rendre visite, un avocat de son choix et son Consulat, sans que les modalités de d'exercice de ce droit soient mentionnées dans quelque instrument juridique. La décision est immédiatement exécutoire et justifie le maintien dans des locaux relevant de cette même autorité jusqu'à l'aboutissement de la mesure ${ }^{31}$.

Le séjour sur le territoire est autorisé pour la durée du visa et, au plus, pour une période de 90 jours. Au-delà, l'étranger doit obtenir une carte d'immatriculation ou de résidence.

28 Loi n 02-03 relative à l'entrée et au séjour des étrangers au Royaume du Maroc, à l'émigration et l'immigration irrégulière promulguée par le Dahir du 11 novembre 2003, Bulletin Officiel du 20 novembre 2002.

29 Voir M. Ben Messaoud, La situation des migrants entre la loi marocaine 02/03 du 11/11/03 et la loi espagnole 14-03 du 20/11/03, in "Lecture de la loi sur la migration », INEJ, Actes du séminaire, journées d'études $n^{\circ} 7$, janvier 2006, p. 49.

30 Son article 58 abroge le Dahir du 15 novembre 1934 réglementant l'immigration en zone française du Maroc, le Dahir du 2 janvier 1940 réglementant le séjour de certaines personnes, le Dahir du 16 mai 1941 relatif aux autorisations de séjour, le Dahir du 17 septembre 1947 relatif aux mesures de contrôle établies dans l'intérêt de la sécurité publique, et le Dahir du 8 novembre 1949 portant réglementation de l'émigration des travailleurs marocains. La rapidité avec laquelle ce texte a été adopté a dû surprendre les rédacteurs du projet de Code du travail qui ont maintenu, bien après l'entrée en vigueur de la Loi 02-03, dans la liste des textes qu'il abroge les Dahirs de 1934 et 1941 abrogés 6 mois plus tôt.

31 Combinaison de l'art. 4 § 5 et de l'art. 34 § 1. 
Pendant tout le séjour il doit être en mesure de présenter à toute réquisition les pièces et documents justifiant la régularité de sa présence.

Mais le droit à un séjour inférieur à 90 jours, ou dont la durée est fixée par le visa, la carte d'immatriculation ou le récépissé en tenant lieu, peut être révoqué «si l'étranger exerce au Maroc une activité lucrative, sans y avoir été régulièrement autorisé, ou s'il existe des indices concordants permettant de présumer que l'intéressé est venu au Maroc pour s'y établir, ou si son comportement trouble l'ordre public $»^{32}$. La mesure peut être assortie de reconduite à la frontière ${ }^{33}$. A moins qu'il soit titulaire de la carte de résidence, l'étranger peut aussi être soumis « en raison de son attitude ou de ses antécédents, à une surveillance spéciale » pouvant donner lieu à l'interdiction de résider dans une ou plusieurs provinces ou préfectures ou à l'indication, à l'intérieur de ces dernières, d'une ou plusieurs circonscriptions de son choix. II ne peut alors se déplacer en dehors de la zone de validité de son titre de séjour sans être muni d'un sauf-conduit délivré par les services de police ou, à défaut, ceux de la gendarmerie royale ${ }^{34}$.

La carte d'immatriculation est délivrée pour une durée de 1 à 10 ans et porte mention, selon les cas, du statut d'étudiant, de la profession autorisée, ou de la qualité de visiteur pour la personne qui vit de ses revenus propres. Lorsqu'à échéance, le titulaire du titre ne demande pas son renouvèlement ou lorsqu'il a été notifié du refus de son octroi ou de son renouvellement, il doit quitter le territoire dans les 15 jours.

Quant à la carte de résidence, elle est attribuée à l'étranger qui réside depuis 4 ans au moins sur le territoire de manière régulière au terme d'un contrôle portant sur les moyens et les conditions d'existence, d'exercice de l'activité professionnelle et le cas échéant, sur tous faits qu'il peut invoquer à l'appui de son intention de s'établir durablement sur le territoire marocain. La loi indique 7 catégories de personnes qui peuvent en bénéficier, mais semble autoriser des dérogations indéterminées ${ }^{35}$. Le rejet de la demande et le retrait de l'autorisation peuvent faire l'objet d'un recours en référé devant le président du tribunal administratif dans le délai de 15 jours. Son introduction «n'empêche pas la prise d'une décision de reconduite à la frontière ou d'expulsion conformément aux chapitres III, IV et V du Titre Premier de la présente loi $»^{36}$. Elle a pour effet de reporter son exécution jusqu'au prononcé de la décision de justice.

La même voie de recours est autorisée à l'encontre de toute décision de reconduite à la frontière. Le président du tribunal administratif saisi est tenu dans ce cas, de statuer dans les 4 jours. Lorsque la mesure a été assortie de la rétention du demandeur dans les locaux de l'autorité de contrôle, c'est à ce magistrat, s'il le souhaite, de se déplacer, au

32 Art. 40.

33 "L'étranger qui a fait l'objet d'une mesure administrative de reconduite à la frontière et qui saisit le président du tribunal administratif, en sa qualité de juge des référés, peut assortir son recours d'une demande de sursis à exécution », Art. 33.

34 Art. 41.

35 Il s'agit de l'époux de Marocain(e), de l'enfant étranger ou apatride d'une mère marocaine, du parent qui a la garde d'un enfant résident au Maroc et naturalisé par le bienfait de la loi, 2 ans au moins avant sa majorité, du conjoint et des enfants mineurs d'un titulaire de la carte de résidence, de ces mêmes enfants à leur majorité, des réfugiés et de l'étranger en situation régulière lorsqu'il établit qu'il a sa résidence habituelle au Maroc depuis 15 ans au moins ou depuis l'âge 10 ans, pendant une durée de 10 ans au moins.

36 Voir art. 20. 
siège de la juridiction la plus proche du lieu de rétention pour auditionner le demandeur. A cette occasion, ce dernier peut lui demander la « communication du dossier, contenant les pièces sur la base desquelles la décision attaquée a été prise », ce qui présume qu'il peut n'en avoir pas pris connaissance jusqu'à ce stade de la procédure. Il peut aussi formuler une demande d'interprète et la désignation d'un avocat commis d'office. On a du mal à imaginer comment ce magistrat peut, dans le délai imparti, satisfaire à ces demandes et se prononcer en audience publique dans les 4 jours. La présence du demandeur à l'audience publique n'est d'ailleurs pas nécessaire dès lors qu'elle lui a été notifiée. Nonobstant la mention faite à la voie de référé, la décision de ce magistrat porte en réalité sur le fond. L'article 24 § 2 déclare que «si la décision de reconduite à la frontière est annulée, il est immédiatement mis fin aux mesures de surveillance prévues à l'article 34 ci-dessous, et l'étranger est muni d'une autorisation provisoire de séjour jusqu'à ce qu'une décision relative à sa situation soit de nouveau prononcée par l'administration ». La sentence est susceptible d'appel devant la juridiction administrative supérieure dans le délai d'un mois. Mais ce recours n'est pas suspensif. L'administration procède donc sans délai à la reconduite à la frontière si la demande en annulation a été rejetée et il lui revient de revoir elle-même sa décision lorsqu'elle est annulée.

Le contrôle de la justice sur les conditions de rétention présente aussi certaines singularités. La loi déclare que le procureur est immédiatement averti de toute décision de maintenir un étranger dans les locaux ne relevant pas de l'ordre judiciaire et pénitencier. La loi l'autorise à s'y rendre pour vérifier les conditions de son déroulement et les registres dans lesquels sont consignées les informations portant sur l'état civil des personnes et les motifs de leur maintien dans ces locaux. Après l'écoulement de 24 heures, l'autorité de contrôle ou le parquet doit saisir le président du tribunal de première instance, en qualité de juge de référé pour décider éventuellement du prolongement de la mesure ou de l'assignation à résidence de la personne concernée pour une période de 15 jours au plus. Ce magistrat peut être saisi ensuite par les mêmes parties d'une demande de reconduction de la décision pour 10 jours encore. Ses décisions sont susceptibles d'appel devant le président de la Cour d'appel qui est tenu aussi de statuer dans les 48 heures.

Le rattachement au droit administratif des attributions dévolues aux autorités de contrôle, comme l'implication formelle du Parquet et des juges civils dans la prise de décision privative de liberté, ne sont pas de nature à présumer de la conformité de telles pratiques avec les droits fondamentaux des migrants. Il suffit de rappeler à ce sujet que I'article 22 de la Convention des Nations Unies sur la protection des travailleurs migrants et des membres de leur famille stipule que la décision d'expulsion prise par l'autorité compétente doit être systématiquement écrite, motivée et notifiée aux intéressés dans une langue qu'ils comprennent. Aux termes du $\S 4$, «En dehors des cas où la décision finale est prononcée par une autorité judiciaire, les intéressés ont le droit de faire valoir les raisons de ne pas les expulser et de faire examiner leur cas par l'autorité compétente, à moins que des raisons impératives de sécurité nationale n'exigent qu'il n'en soit autrement. En attendant cet examen, les intéressés ont le droit de demander la suspension de la décision d'expulsion».

La voie pénale accentue la dissuasion et l'efficacité des mesures qualifiées d'administratives par la loi. Le Parquet peut à tout moment recourir notamment à l'article 42 pour écarter toute velléité de contestation des décisions de l'autorité de contrôle. Le texte sanctionne la tentative de pénétration sur le territoire national, comme l'irrespect des prescriptions légales ou réglementaires relatives au séjour et à sa prolongation au-delà de 
la durée autorisée, d'une amende de 2000 à 20000 dirhams et d'un emprisonnement de 1 à 6 mois, ou de l'une de ces deux peines seulement. Toutefois, l'autorité administrative peut, eu égard aux impératifs découlant de la sécurité et de l'ordre public, expulser l'étranger vers le pays dont il est ressortissant ou vers un autre pays, selon son souhait.

La résidence sur le territoire sans être titulaire de la carte d'immatriculation ou de résidence est punie d'un mois à un an d'emprisonnement, et d'une amende pouvant atteindre 30000 dirhams, tandis que la soustraction à une décision d'expulsion vaut à son auteur 2 années de prison. La plupart de ces infractions et de nombreuses autres sont punies du double en cas de récidive.

La procédure pénale prévoit bien l'assistance judiciaire et la présence d'un interprète dans la phase de jugement lorsque le prévenu parle une langue inaccessible au magistrat. En revanche, rien de tel n'est prévu dans la phase de l'enquête policière et de la garde à vue. Les prévenus sont de ce fait acculés à signer des procès-verbaux rédigés en arabe et ne mentionnant pas les questions posées. Comme ces documents font foi jusqu'à inscription de faux, les chances d'un procès équitable paraissent d'autant plus limitées que de nombreux tribunaux manquent cruellement de moyens ${ }^{37}$.

On peut déduire de ce survol de la Loi 02/03 que ses dispositions confèrent un pouvoir discrétionnaire quasi illimité à l'autorité de contrôle pour déclarer un étranger en situation irrégulière et décider à son encontre, de manière expéditive, des mesures de rétention, de reconduite à la frontière ou d'expulsion, voire une action publique aboutissant à son emprisonnement. Un tel dispositif est des plus dissuasifs à l'égard des migrants qui envisagent de s'installer au Maroc pour y travailler et y vivre. Il est en revanche de peu d'effet sur tous ceux qui inscrivent leur projet d'émigration dans l'irrégularité depuis la pénétration sur le territoire national et dont l'identification est rendue difficile en conséquence. Or, c'est à l'encontre de cette migration irrégulière que la stratégie européenne a été mise en place et étendue aux pays de voisinage.

\section{B - UN DROIT DU TRAVAIL EN RETRAIT PAR RAPPORT AUX BESOINS D'INTÉGRATION DES IMMIGRÉS}

La ratification de la Convention sur la protection des droits des travailleurs migrants et des membres de leur famille n'a manifestement pas retenu l'attention des rédacteurs du Code du travail, entré en vigueur en 2004, pas plus d'ailleurs que l'entrée en vigueur quelques mois plus tôt de la Loi 02/03.

Le Chapitre IV du livre IV du Code du travail a ainsi repris des textes antérieurs les dispositions embryonnaires qui confèrent à l'autorité gouvernementale chargée du travail la mission d'autoriser l'emploi salarié des travailleurs étrangers, en apposant son visa sur leur contrat de travail ${ }^{38}$. La Loi 19-12 relative aux travailleuses et travailleurs domestiques ${ }^{39}$ a étendu l'application de la règle à cette catégorie de salariés. Toutefois, les pouvoirs

37 Voir N. Khrouz, «De la respécification de la notion de transit », in Les migrants au Maroc: https:// books. openedition.org/cjb/865

38 Le modèle antérieur du contrat de travail de l'étranger était régi par l'arrêté du Ministre du travail et des affaires sociales $n^{\circ} 714.64$ du 25 décembre 1964, fixant le modèle de contrat prévu au Dahir du 15 novembre 1934 relatif à l'immigration.

39 Promulguée par le Dahir du 10 aout 2016, B.0 n6610 du 5 octobre 2016, p. 1038. 
qu'exerce l'administration du travail à ce titre n'ont qu'une faible emprise sur leur droit au séjour sur le territoire (1). La situation des travailleurs étrangers non-salariés semble encore plus confuse en l'absence d'un régime juridique déterminant clairement dans quelles conditions ils sont admis à exercer des activités professionnelles (2).

\section{1 - L'emploi salarié des étrangers}

L'article 516 du Code du travail dispose que « tout employeur désireux de recruter un salarié étranger doit obtenir une autorisation de l'autorité gouvernementale chargée du travail. Cette autorisation est accordée sous forme de visa apposé sur le contrat de travail. La date du visa est la date à laquelle le contrat de travail prend effet. Toute modification du contrat est également soumise au visa mentionné au premier alinéa du présent article. L'autorisation peut être retirée à tout moment par l'autorité gouvernementale chargée du travail». La disposition suivante ajoute que «le contrat de travail des étrangers doit être conforme au modèle fixé par l'autorité gouvernementale chargée du travail ».

En vertu de ces dispositions, l'autorité chargée du travail a adopté l'arrêté du 9 février $2005^{40}$, dans la continuité de la pratique administrative antérieure qui aboutissait à transformer tous les engagements contractuels en CDD en accordant un visa pour un an au plus. Le dispositif a été modifié par l'arrêté du 30 octobre $2018^{41}$ et finalement remplacé en vertu de l'arrêté du 19 avril $2019^{42}$ actuellement en vigueur.

Tous ces textes sont d'une sobriété extrême et reportent une partie de leurs prescriptions sur le modèle de contrat qui leur est annexé. C'est à travers la liste des documents requis qu'on présume des critères retenus. Leur liste est actuellement fixée par une décision du Ministère du travail ${ }^{43}$.

Le nouveau modèle de contrat apporte une modification substantielle à la pratique antérieure en prévoyant la possibilité d'opter pour un CDI ou un CDD. Rien n'indique cependant si l'administration peut accorder d'office une durée moins longue, obligeant le requérant à procéder au renouvellement de la demande à l'échéance, comme elle l'a fait jusqu'à présent, ou à s'en tenir à l'option choisie par l'employeur pour accepter ou refuser sa demande. C'est d'ailleurs seulement au moyen d'un document d'information publié sur le site web du ministère que l'on peut glaner des informations sur la pratique administrative suivie $^{44}$.

Une procédure commune dématérialisée administrée par le Département chargé du travail aboutit à l'apposition du visa d'autorisation sur le contrat de travail. Elle est

40 Arrêté du Ministre de l'emploi et de la formation professionnelle n 350-05 du 9 février 2005 fixant le modèle du contrat de travail réservé aux étrangers, Bulletin Officiel n 5540 du 5 juillet 2007, p. 908.

41 B. $0 n^{\circ} 6736$ du 20-12-2018, p. 1952.

42 B. $0 n^{\circ} 6786$ du 13 juin 2019 (éd. en arabe).

43 Décision du MTIS n 1/Taechir/2019 du 1er juillet 2019.

44 «Procédure d'emploi des salariés étrangers au Maroc » mise à jour en juillet 2019. 
précédée, sauf pour les catégories de travailleurs qui en sont expressément exemptées ${ }^{45}$, d'une procédure d'investigation qui se déroule auprès de l'ANAPEC en vue de l'obtention d'une attestation d'activité ${ }^{46}$. L'intervention de cet organisme dans la procédure n'a pas été prévue par le Code du travail et ne figure pas non plus dans les attributions légales de cet établissement public ${ }^{47}$. Comme l'administration chargée du travail hérite en la matière d'une compétence large lui permettant même de retirer son autorisation à tout moment, sans avoir à motiver cette décision ${ }^{48}$, on peut admettre qu'elle peut valablement externaliser une partie - ou la totalité - de la procédure à I'ANAPEC, ou à une toute autre personne morale, notamment lorsqu'elle est placée sous sa tutelle, comme c'est le cas en l'occurrence. Le mandat qui lui a été confié consiste à vérifier s'il n'existe pas, parmi les compétences nationales se trouvant sur le marché de l'emploi, des profils correspondant au poste à pourvoir. A cet effet, elle instruit le dossier de la demande, qui doit comprendre notamment les justificatifs, le modèle de l'annonce à publier et le reçu de paiement de la prestation ${ }^{49}$. Une procédure simplifiée dispense de la publication de l'appel à candidatures lorsqu'il s'agit de postes de responsabilité de haut niveau ${ }^{50}$, de profils présumés rares et non disponibles au Maroc ${ }^{51}$, et de renouvellement de l'attestation pour le même poste ou un poste sur lequel le salarié a été promu.

45 En l'occurrence : 1) le conjoint de Marocain, 2) descendant de mère marocaine, 3) natif du Maroc, 4) regroupement familial, 5) associé, 6) gérant, 7) fondé de pouvoir, 8) détachement, 9) séjour de plus de 10 ans, 10) mission de courte durée, 11) régularisation exceptionnelle, 12) jeune professionnel, 13) Casa Finance City, 14) offshorig, 15) convention d'établissement, 16) sportifs, 17) réfugiés et apatrides, 18) catégories particulières. C'est à travers l'examen des pièces requises pour chaque catégorie qu'on dégage plus précisément les conditions requises.

46 Jusqu'aux aménagements introduits en 2019, les exemptions étaient formellement limitées aux: étrangers nés au Maroc ou descendant d'une mère marocaine à condition qu'ils soient résidants depuis six mois au moins; époux (ses) étranger (es) de Marocains (es); détaché (e) s pour une période limitée auprès d'entreprises étrangères ou de leurs filiales qui réalisent des projets au Maroc; personnes mandatées par un employeur étranger dans le cadre de la coopération, pour une période de six mois au plus; réfugiés politiques et les apatrides.

47 On relève toutefois qu'en vertu de l'art. 3, l'ANAPEC a pour mission de contribuer à l'organisation et la mise en œuvre des programmes de promotion de l'emploi qualifié, décidés par les pouvoirs publics. Voir Loi n 51-99 portant création de l'Agence nationale de promotion de l'emploi et des compétences, promulguée par le Dahir du 5 juin 2000, B.0 n4804 du 15/06/2000, p. 460.

48 Voir art. 516 du Code.

49 Les frais sont de 5000 dh pour la demande requérant la publication de l'annonce, et de 1500 dh pour chaque demande supplémentaire, dans la limite de 5 par poste et pour une même entreprise au cours de l'année. Ils sont de 1500 par demande lorsque l'annonce n'est pas requise.

50 Liste A1 comprenant PDG, hauts personnels de direction, premier responsable d'une entreprise multinationale ou entreprises employant plus de 50 travailleurs et ayant investi un million de dh au moins, $2^{\text {ème }}$ responsable d'établissement de 500 salariés, responsable d'ONG avec 3 ans d'expérience au moins, et responsable d'une succursale avec 2 ans d'expérience dans la maison mère.

51 La liste comprend plus de 20 profils, allant des directeurs de programmes Radio et TV, aux ingénieurs, architectes et commandants de bord expérimentées jusqu'aux majordomes, entraineurs de chevaux, et éleveurs de dromadaires. 
Le rapprochement de ce dispositif avec celui du contrôle sur l'immigration laisse à penser que le profil du travailleur visé est celui qui se trouve à l'étranger et que l'entreprise concernée cherche à engager, en justifiant de l'obtention d'un visa d'entrée pour le travail, puis en engageant la procédure réglementaire pour obtenir le visa du contrat de travail de l'étranger. Les dispositions en vigueur prévoient d'ailleurs l'obligation pour l'employeur demandeur de supporter les frais de rapatriement en cas de refus de visa du travail. Elles demeurent toutefois silencieuses sur le rapatriement à l'extinction du contrat, soit par l'arrivée de son terme, soit par rupture anticipée.

On relève également que les pouvoirs attribués aux autorités de contrôle de l'immigration, en matière d'octroi et de retrait des titres de séjour, s'exercent abstraction faite du régime de travail. C'est d'ailleurs ce qui semble constituer le rappel de l'amendement introduit en 2018, sur l'arrêté du ministre du travail de 2005, qui énonce: "s'appliquent à ce contrat, toutes les dispositions, en la matière, en vigueur dans le Royaume du Maroc $»^{52}$.

Une fois admis au travail, le salarié étranger accède aux mêmes droits et assume les mêmes obligations que les Marocains, à trois exceptions près. En matière de représentation collective, les étrangers ne sont admis ni à la direction des syndicats, ni à se présenter aux élections des représentants au sein des établissements. Au plan des droits individuels, la pratique administrative qui consistait jusqu'à présent à leur accorder des visas sur les contrats de travail pour une durée d'un an au plus, empêche que la relation de travail relève des dispositions protectrices en matière de licenciement. En dehors de la relation salariée, le droit applicable aux travailleurs étrangers demeure relativement confus en raison du silence de la loi sur l'exercice par les étrangers des activités non réglementées et des conditions prescrites pour l'exercice des différentes professions libérales.

\section{2 - Le travail non salarié des immigrés}

La règle de base interdit à tout étranger pourvu d'un visa de séjour n'excédant pas 3 mois d'exercer une activité lucrative, sans y être autorisé. En cas de violation de cette prohibition et chaque fois que des indices concordants permettant de présumer que le visiteur est venu au Maroc pour s'y établir, le titre de séjour peut être annulé53. Mais telle que formulée par l'article 40, la mesure n'écarte pas la possibilité d'exercer une activité lucrative, même si le visa est de très courte durée, pourvu que son titulaire obtienne l'autorisation à cet effet. Reste à déterminer le type d'autorisation dont il s'agit, l'autorité habilitée à la délivrer et les modalités de la demande.

La deuxième règle concerne l'étranger qui apporte la preuve qu'il peut vivre de ses seules ressources. La carte d'immatriculation qui lui est délivrée porte la mention de «visiteur». Pour l'obtenir, il doit prendre «l'engagement de n'exercer au Maroc aucune activité professionnelle soumise à autorisation ». A ce niveau aussi, l'interdiction du travail n'est pas formulée de manière absolue. Telle qu'elle est rédigée, elle signifie plutôt que

52 Voir art. 3 de l'arrêté du Ministre du travail et de l'insertion professionnelle du 30 octobre 2018 modifiant l'arrêté du MEFP du n³50-05 du 9 février 2005, fixant le modèle de contrat de travail réservé aux étrangers, B.0. n 6736 du 20/12/2018, p. 1952. La disposition est reprise à l'article 2 de l'arrêté de 2019.

53 Art. $40 \S 2$. 
l'interdiction porte seulement sur l'exercice d'activités relevant d'une autorisation, soit pour tous, soit pour des catégories déterminées de travailleurs, notamment les étrangers ${ }^{54}$.

Le même article 13 régit le séjour motivé par la formation. Il déclare que «la carte d'immatriculation délivrée à l'étranger qui établit qu'il suit au Maroc un enseignement ou qu'il y fait des études et qui justifie de moyens d'existence suffisants, porte la mention "étudiant" ». Cette règle n'évoque pas l'exercice d'un travail par les apprenants. II n'est nullement indiqué que ce statut empêche l'exercice par le migrant étudiant de toute activité rémunérée, fut-elle liée à sa formation ou de très courte durée.

Le paragraphe 3 de ce même article 13 est le seul qui traite de l'autorisation de séjour pour le travail. Mais il est aussi sommaire que les précédents. II dispose seulement que «la carte d'immatriculation délivrée à l'étranger désirant exercer au Maroc une activité professionnelle soumise à autorisation et qui justifie l'avoir obtenue, porte la mention de cette activité ». La mesure ne s'applique qu'à l'exercice d'une activité soumise à autorisation et c'est seulement au terme de son obtention que la carte d'immatriculation peut être délivrée au travailleur migrant avec mention de l'activité autorisée.

La question reste ouverte dans les trois situations au sujet de l'exercice des activités qui ne sont pas explicitement soumises à autorisation; en l'occurrence, celles qui ne relèvent ni du droit du travail, ni des professions réglementées. La plupart des professions réglementées réservent leur exercice aux Marocains et prévoient une procédure d'inscription obligatoire. $C^{\prime}$ est le cas des experts comptables ${ }^{55}$, notaires ${ }^{56}$, avocats ${ }^{57}$, ingénieurs topographes ${ }^{58}$, médecins ${ }^{59}$, pharmaciens ${ }^{60}$ et architectes ${ }^{61}$. D'autres textes sont moins explicites, soit en ce qui concerne la condition de nationalité, soit l'inscription obligatoire dans l'ordre, tout en soumettant l'accès à la profession à une procédure obligatoire d'autorisation, diligentée auprès du Secrétariat général du gouvernement ${ }^{62}$. Le site web de ce dernier place à maintes reprises le texte de la Loi 02-03 parmi les dispositifs régissant les professions concernées, comme pour garder une certaine réserve sur leur compatibilité.

54 Art. $13 \S 1$.

55 Voir art. 20 de la Loi 15-89 promulguée par le Dahir du 8 janvier 1993 portant réglementation de la profession d'expert-comptable.

56 Voir art. 3 de la Loi 32-09 promulguée par le Dahir du 22 novembre 2011 relative à la profession de notaire.

57 Voir art. 5 de la Loi 28-08 promulguée par le Dahir du 20 octobre 2008 relative à la profession d'avocat.

58 Voir art. 22 de La loi 30-93 promulguée par le Dahir du 25 février 1994 relative à l'exercice de la profession d'ingénieur topographe.

59 Voir art. 4 de la Loi 131-13 promulguée par le Dahir du 10 février 1915 relative à l'exercice de la médecine.

60 Voir art. 93 de la Loi 17-04 portant Code du médicament et de la pharmacie, promulguée par le Dahir du 22 novembre 2006.

61 Voir art. 4 de la loi 016-89 promulguée par le Dahir du 10 septembre 1993 relative à l'exercice de la profession d'architecte.

62 Voir notamment: art. 20 de la loi 43-13 promulguée par le Dahir du 22 juin 2016 relative à l'exercice de la profession d'infirmier; art. 4 de la Loi 12-01 relative aux laboratoires privés de biologie médicale, promulguée par le Dahir du 3 octobre 2002; art. 4 de la Loi 07-05 relative à l'Ordre national des médecins dentistes; art. 3 du décret du 9 juin 1995 pris pour l'application du Dahir portant loi du 6 octobre 1993 relatif à l'Ordre national des vétérinaires. 


\section{III - UNE GOUVERNANCE MIGRATOIRE DANS LA DÉPENDANCE}

Tel qu'il a été présenté dans les développements qui précèdent, le droit marocain de la migration exprime certaines tensions entre les objectifs sécuritaires qu'il partage avec I'Union Européenne (A) d'une part, et l'approche par les droits qu'il s'est engagé à adopter lors de la ratification de la Convention des Nations Unies; droits invoqués dans la politique qu'il entend développer, notamment en direction des pays subsahariens dans le domaine de la migration (B).

\section{A - UNE POLITIQUE MIGRATOIRE SOUS EMPRISE EUROPÉENNE}

L'existence des enclaves de Ceuta et Melilla sur la rive sud de la Méditerranée comme la proximité de l'Europe, distante de $14 \mathrm{~km}$ seulement dans le détroit de Gibraltar, donnent forcément lieu à une coopération sécuritaire régulière en matière de contrôle de la mobilité frontalière avec l'Espagne. Mais à mesure qu'une politique commune européenne s'est mise en place pour fortifier les frontières extérieures de l'Europe, les partenaires du sud ont été appelés à coopérer à sa mise en œuvre, tant avec les pays de voisinage que dans le cadre global euro-méditerranéen.

Le volet de l'assistance militaire et policière se traite naturellement dans la discrétion. II s'exprime au mieux par l'annonce des crédits qui lui sont consacrés et par les statistiques qui affichent les succès remportés, en termes de tentatives avortées de migration clandestines et de réduction du volume des arrivées sur le territoire européen. C'est la même discrétion qui prévaut sur le cadre institutionnel et opérationnel dont a été dotée la Direction Générale de la Sûreté Nationale pour assurer les multiples missions conférées par la Loi 02/03.

En revanche, au niveau du Ministère de l'intérieur, un amendement a été introduit dès l'entrée en vigueur de cette loi sur son organigramme ${ }^{63}$ pour conférer à la Direction générale des affaires intérieures la mission de "définir les orientations du ministère en matière de coopération internationale et organiser la coordination de l'activité de tous les services chargés de la sécurité et de l'ordre publics. A cet effet, elle assure le suivi et l'évaluation permanents de la situation sécuritaire au Royaume $\|^{64}$. Le même texte porte création de deux nouvelles directions chargées de la migration, du contrôle des frontières et de la coopération internationale. La première s'occupe particulièrement de «la mise en œuvre opérationnelle et du suivi de la stratégie nationale en matière de lutte contre les réseaux de trafic des êtres humains et du contrôle des frontières ". La seconde hérite des attributions antérieures de la division chargée de la lutte contre la drogue, auxquelles s'ajoutent les activités de coopération et de gestion des flux migratoires.

Il faut rappeler à ce sujet que les négociations sur « l'ordre migratoire » engagées entre le Maroc et l'Union Européenne en 1998 avaient pour objet la lutte contre la migration clandestine, formulée dans une approche de « régulation et structuration de la circulation des personnes entre le Maroc et l'Union Européenne ». C'est dans ce cadre qu'il avait été décidé de désigner l'ANAPEC comme partenaire marocain officiel pour la gestion des

63 Décret du 27 décembre 2004 modifiant et complétant le décret du 15 décembre 1997 fixant les attributions et l'organisation du ministère de l'Intérieur, B.O nº 5280 du 6 janvier 2005, p. 74.

64 Art. 1 portant modification de l'art. 3 du décret de 1997, traduction non officielle. 
offres d'emploi européennes, de créer en son sein une division internationale, de la doter d'une antenne à Bruxelles et de quatre Agences à l'international ${ }^{65}$.

Mais si la coopération dans la lutte contre la migration irrégulière ne rencontre pas du côté européen des limites, telles que celles qui découlent pour le Maroc de la Convention des Nations Unies sur la protection des droits des travailleurs migrants et des membres de leur famille, les deux parties partagent les mêmes engagements s'agissant du respect des droits des réfugiés, conformément aux dispositions de la Convention de Genève de 1951 et à son protocole de 196766. Or à ce sujet aussi, I'Union Européenne a développé une stratégie visant à reporter sur les pays voisins le traitement des demandes d'asile. Plus qu'une externalisation de ce service, il s'agit en fait, grâce à la médiation du HCR, de leur reconnaitre sur place le statut de réfugié.

Jérome Valluy relève que l'idée d'externalisation de l'asile est présente dans les rouages administratifs et politiques de l'Union depuis les années 1990. Elle s'est matérialisée en 1998 par un « Document de stratégie sur la politique de I'UE en matière de migrations et d'asile», diffusé par la présidence autrichienne de I'Union. Un «Groupe de Haut niveau asile-migration » a été constitué pour finaliser l'inclusion de l'Organisation Internationale de la Migration (OIM) et du Haut-Commissariat aux Réfugiés (HCR) dans le processus. Une ligne a été inscrite depuis 2001 sur le budget afin d'instaurer « une coopération technique et financière dans le domaine de l'asile et des migrations, en faveur des pays tiers ».

Le Premier Ministre britannique Tony Blair a été le promoteur en 2003 d'une proposition tendant à créer, dans les pays voisins de l'UE, des camps d'enfermement des exilés déjà arrivés et souhaitant s'installer en Europe ${ }^{67}$. La désinscription de cette proposition de l'ordre du jour du Sommet de Thessalonique (2 juin 2003) n'a pas empêché le Royaume-Uni, avec l'appui d'autres pays, d'expérimenter le projet dans certains pays, en abandonnant l'idée de camps tout en impliquant le HCR. Une dotation d'un million d'euros, financée à hauteur de $80 \%$ par l'UE et à $20 \%$ par les pays, a été allouée à cet effet et ciblait particulièrement le Maghreb. Son déblocage se destinait formellement à la «constitution d'un système national d'asile ». Il est difficile de ne pas voir dans le redéploiement du HCR et de I'OIM au Maroc un rapport avec ces agendas ${ }^{68}$.

En réalité, depuis l'indépendance, le HCR a été présent au Maroc via un Bureau ouvert à Casablanca, dirigé par un représentant honoraire assisté d'un agent et d'un chauffeur. Jusqu'à 2004, la réception et l'acheminent des dossiers au HCR avaient abouti à l'attribution du statut à 272 réfugiés. Sans préalable, cette représentation a été transférée en 2004 à

65 Un financement a été prévu par cette même occasion réaliser une étude pour déterminer les conditions de viabilité du retour volontaire des MRE pour ceux qui souhaitent la réintégration professionnelle dans leur pays et la création d'un centre documentaire sur les voies de la migration légale vers l'Europe.

66 Résolution 2198 (xxi) de l'Assemblée générale.

67 Voir J. Valluy, "Le HCR au Maroc: acteur de la politique européenne d'externalisation de l'asile », in L'Année du Maghreb, III, 2007, Editions CNRS, p. 547.

68 Selon une dépêche de l'AFP, le Commissaire Européen à l'intérieur a déclaré a propos de cette enveloppe: "concrètement, le Haut-commissariat aux réfugiés des Nations-Unis (HCR) va mener ce projet, comme il le fait déjà dans de nombreux pays, avec l'espoir que cela sera suffisant pour inciter les demandeurs d'asile à rester sur place plutôt que de traverser la Méditerranée ", AFP, "L'UE engage un débat confus sur la réponse aux naufragés de la Méditerranée » par Fabrice Randoux, Scheveningen (Pays-Bas), 1/10/2004. 
Rabat, son titulaire remercié et de nouveaux bureaux dotés de moyens et d'équipements conséquents mis à sa disposition. C'est le PNUD qui a offert ses services à cet effet dans la mesure où le HCR n'avait pas encore conclu de convention de siège avec le Maroc.

S'en est suivie une activité intense de ses services tant en direction des ONG que des candidats au statut de réfugiés. La délivrance de récépissés de dépôt de leur demande qui leur confère une protection à l'égard des autorités marocaines n'a pas tardé à produire un effet d'appel sur de nombreux migrants et le mécontentement des autorités marocaines, qui s'en trouvaient marginalisées. Ce n'est qu'en juillet 2007 qu'un accord de siège a été signé avec le Maroc.

Le droit interne applicable en la matière demeure constitué par le Décret royal du 29 août 1957, fixant les modalités d'application de la Convention relative au statut des réfugiés de 1951, qui prévoit l'installation d'un Bureau des Réfugiés et Apatrides (BRA) au sein du Ministre des Affaires Etrangères et d'une Commission de recours comprenant le Ministre de la Justice ou son représentant, le Ministre des Affaires Etrangères ou son représentant, ainsi que le Représentant de I'UNHCR auprès du Gouvernement du Maroc. La mise en place d'une procédure qui tient compte des apports du protocole de 1967 constitue encore un enjeu de négociation.

On peut lire sur le site du HCR: «Dans l'attente de l'établissement d'un système national d'asile, le HCR continue d'enregistrer les demandeurs d'asile et d'intervenir en cas d'arrestation, de détention ou de tout autre problème de protection. Le HCR travaille étroitement avec les autorités marocaines afin de garantir aux réfugiés et demandeurs d'asile une protection optimale. Plus spécifiquement, le HCR soutient le Bureau des Réfugiés et des Apatrides du MAEC et la Commission interministérielle en charge du processus de régularisation. Cette Commission ad hoc mène des auditions des réfugiés reconnus par le HCR, permettant à ceux-ci de recevoir par la suite une carte de réfugié des autorités, excepté pour les Syriens qui n'obtiennent qu'un récépissé ».

L'activité du HCR prend aussi la forme d'assistance aux postulants au moyen des services d'avocats et de partenariats avec des ONG nationales et étrangères pour développer des activités localisées de référencement, d'éducation, de santé et d'aide financière.

Un déploiement similaire a été réalisé par I'OIM ${ }^{69}$ dès 2001. En 2006 elle s'est installée officiellement avec des antennes dans plusieurs villes. Ses activités portent sur le rapatriement des migrants vulnérables, tant nationaux qu'étrangers, le soutien de leur réintégration dans les pays d'origine, le relèvement des capacités en matière de gestion des flux migratoires mixtes et d'intégration socioprofessionnelle des migrants réguliers ainsi que sur «l'assistance humanitaire aux migrants en situation irrégulière au Maroc ».

69 Cette organisation a vu le jour en 1951 sous forme d'un Comité intergouvernemental de pays européens ayant pour mission de faciliter le transport et la réinstallation de près d'un million de déplacés de la guerre. Ayant son siège à Genève, elle est demeurée active dans des activités de logistique tout en développant un savoir-faire sur les questions migratoires. L'augmentation massive des flux et le déploiement de politiques restrictives d'admission des migrants et des réfugiés dans les pays riches lui ont permis de devenir un acteur incontournable dans les débats sur les questions migratoires et un opérateur central dans leur financement. Son budget avoisine 2 milliards de dollars. Depuis 2016, elle a le statut d'organisation apparentée l'ONU. 
Cette présence est soutenue et relayée par la coopération de I'UE. Elle s'est manifestée clairement à travers MEDA $\mid{ }^{70}$. L'adoption en 2011 de la stratégie du partenariat pour la mobilité a renouvelé la démarche «venant ainsi compléter les objectifs distincts et plus vastes de la politique extérieure et de la coopération au développement de l'UE ».

Les activité du HCR, de l'OIM, de I'Union Européenne et des différentes chancelleries occidentales au Maroc convergent sur les objectifs de rapatriement des migrants, de sanctuarisation du territoire européen et d'assistance humanitaire aux migrants irréguliers avec le dessein d'intégration ou de rapatriement. Alors que les effectifs des migrants irréguliers subsahariens augmentent au rythme des succès de la coopération sécuritaire, le Maroc est poussé à reconnaitre qu'il serait devenu un pays de destination pour ceux qui s'y trouvent piégés. Les événements dramatiques de Ceuta et Melilla en 2005 ont établi les limites d'une politique migratoire portée principalement par les objectifs sécuritaires. Faute de réponse à la mesure des origines d'un mal qui porte de plus en plus de jeunes tant marocains que subsahariens à prendre tous les risques pour arriver à l'autre rive de la Méditerranée, le Maroc cherche les compromis soutenables pour une gouvernance migratoire qui satisfait à la fois ses partenaires européens et africains, sans lui imposer des coûts économiques et politiques excessifs.

\section{B - UNE GOUVERNANCE MIGRATOIRE DE COMPROMIS}

Le droit applicable à la condition des immigrés n'a pas connu véritablement la mise à jour qu'impose la ratification de la Convention de 1990 précitée ${ }^{71}$. Sa réforme par la Loi 02-03 sur les droits des étrangers soulève au contraire de nouveaux problèmes, tant pour la reconnaissance des droits immigrés que pour leur protection ${ }^{72}$. Les commentaires suscités au Haut-Commissariat des Nations-Unies à l'occasion de l'examen du rapport initial du Maroc sur ladite Convention donnent la mesure de ce déni de droits. L'état d'abandon et de transhumance entretenu par les déplacements collectifs forcés de dizaines de milliers subsahariens en est l'expression parlante, en permanence véhiculée par l'actualité internationale. L'Europe qui propose à ses partenaires du sud une approche globale de la migration, de la mobilité et du développement, comme le Maroc qui développe à l'égard des pays africains une politique de coopération sud/sud, en assument - au moins partiellement - une part importante de responsabilité, sinon juridique du moins politique. Il est dès lors important que la responsabilité partagée qu'ils invoquent s'exprime dans une nouvelle politique soucieuse des droits fondamentaux des migrants. Mais tant que le système global de régulation des flux migratoires génère aux frontières extérieures du monde riche une transhumance migratoire régulière, cette politique ne peut se poursuivre que par une étroite prise en charge par les pays concernés.

70 La coopération entre le Maroc et I'UE s'inscrit dans le cadre du partenariat euro-méditerranéen lancé à Barcelone en 1995 et basé sur l'accord d'association, entré en vigueur en 2000. Le Règlement MEDA II était le principal instrument de financement du partenariat pour la période 2000-2006, mais il a été remplacé par l'instrument européen de voisinage et de partenariat, IEVP, pour la période 2007-2013.

71 Sur la non-conformité du droit positif avec les prescriptions de la convention des N.U, voir A. Bouharrou et R. Filali Meknassi, « Rapport sur l'état des lieux juridique des droits des travailleurs migrants au Maroc », BIT, Projet AMEM, titre I, chap. 1, Rabat, octobre 2019, p. 220.

72 Idem, titre II, chap. 1. 


\section{1 - Genèse d'une nouvelle politique globale migratoire}

Le rapport publié par le Conseil Consultatif des droits humains en 2007, suite à l'enquête réalisée sur les évènements de Ceuta et Melilla de 2005, dégage clairement le sentiment généralisé d'insatisfaction à l'égard d'une politique migratoire qui impose le recours à la répression systématique des migrants en situation irrégulière pour sécuriser les frontières extérieures de l'Europe. Mais la fermeté de ses termes n'est sans doute pas étrangère au fait que la partie européenne était déjà arrivée au même constat, et proposait une nouvelle approche globale des migrations fondée sur un partage des responsabilités entre les Etats d'origine, de transit et de destination ${ }^{73}$.

Les termes de l'évaluation de la situation par le CNDH sont tranchants: "Le Maroc, en plus de ses propres ressortissants candidats à l'émigration, est devenu, à l'évidence, un lieu de passage pour nombre de candidats à l'émigration, provenant de nombreux pays africains ou autres. Par la grâce et l'alliance de la géographie et de l'histoire, notre pays est devenu l'ultime frontière du sud de l'espace Schengen. II constitue, avec le détroit de Gibraltar et les deux villes de Ceuta et Melilla, le plus infime lieu de passage entre l'Europe forteresse et l'Afrique détresse. Les assauts, les conditions de leurs constitutions ainsi que celles de leurs traitements par les pouvoirs publics marocains et espagnols, illustrent, jusqu'à la caricature, les conséquences du raisonnement répressif par lequel l'Europe a choisi de traiter la question des migrations (...). Au mépris de leurs engagements internationaux, en particulier, ceux relatifs aux droits des réfugiés, les Etats membres exercent des pressions importantes sur les pays de transit comme le Maroc, pour que tout soit mis en œuvre pour contrôler, parer, chasser et refouler les populations migrantes. Si l'Europe forteresse se construit à coups de politique restrictive, les pays riverains, comme le Maroc, sont devenus au fil du temps, comme d'énormes salles d'attente. Ainsi, dans les forêts et les banlieues des grandes villes du nord marocain, des milliers de migrants subsahariens ont pris l'habitude d'attendre, dans des conditions inhumaines, les possibilités, de plus en plus illusoires et aléatoires, de franchir le mur de barbelés, à défaut de franchir le détroit et sa grande bleue $»^{74}$.

Les conséquences du recours à la voie répressive y sont aussi évoquées. Le Rapport note que le Maroc s'est employé à lutter contre toutes les formes de cette immigration pour garantir la protection de sa sécurité intérieure, et contribuer à préserver la sécurité au plan régional et international (...) « avec les conséquences regrettables qui peuvent entacher

73 L'approche est définie comme la dimension extérieure de la politique de l'Union européenne en matière de migrations. Elle couvre l'ensemble des questions ayant trait aux migrations d'une manière exhaustive et équilibrée (migration légale, lutte contre l'immigration illégale, lien entre migration et développement et dimension extérieure de l'asile). A la suite de l'évaluation dont elle a été l'objet dans la communication de la Commission présentée le 18 novembre 2011 au Conseil, ce dernier a décidé de l'adapter aux nouveaux besoins migratoires avec le titre «La nouvelle approche globale de la question des migrations et de la mobilité ».

Pour sa mise en œuvre, I'Union Européenne s'appuie sur des processus régionaux (principalement processus dit «de Rabat» avec l'Afrique de l'ouest, et processus dit " de Prague » à l'est) et 4 instruments: les missions migratoires, les plateformes de coopération, les profils migratoires et les partenariats pour la mobilité. Voir: https://eur-lex.europa.eu/LexUriServ/LexUriServ. do?uri=COM:2011:0743:FIN:FR:PDF

$74 \mathrm{CNDH}$, Rapport sur l'établissement des faits relatifs aux événements de l'immigration illégale, Evènements de de Ceuta et Melilla durant l'automne 2005: https://cndh.org.ma/IMG/pdf/CCDH BLEU 17X24 FR.pdf 
des opérations sécuritaires, prises à défaut par l'ampleur des assauts sur les frontaliers, leur imprévision et parfois, l'impréparation des personnels ».

Les principales recommandations ciblent les réfugiés et sonnent comme un message au HCR pour engager une meilleure coopération après la signature de l'accord de siège ${ }^{75}$. Quant au traitement de la migration et de l'immigration irrégulières, il se contente de dénoncer le cloisonnement des actions, la situation des dizaines de milliers de migrants et de plaider " pour une harmonisation entre tous les acteurs qui interviennent dans le domaine des migrations aussi bien pour les flux de nos propres ressortissants que pour ceux qui viennent de l'extérieur. Il y voit l'avantage de la synergie, de l'économie de moyens et surtout le développement d'une expertise dotée d'une vision globale».

Le même Conseil qui a accédé en 2011 au rang d'institution constitutionnelle sous la dénomination de Conseil national des droits humains a adopté ces recommandations, six années plus tard, au terme d'une concertation élargie dont la sévérité du constat a empêché sa publication intégrale. Mais ses recommandations en faveur d'une nouvelle stratégie nationale de l'immigration et de l'asile ont immédiatement trouvé une voie royale pour donner le change aux critiques soulevées par la discussion du rapport initial du Maroc sur les droits des migrants, et pour envoyer un message fort au pays africains ${ }^{76}$.

\section{2 - Les perspectives d'une nouvelle politique d'immigration et d'asile}

Le rapport du CNDH récapitule le processus de coopération en partant de l'entrée en vigueur de la Loi 02-03. II fait mention de la conclusion de l'accord de siège avec le HCR et de l'engagement, avec le soutien de I'Union Européenne, d'une politique de contrôle des tentatives d'émigration avec un réel succès (comme en témoignent les chiffres d'interpellation et les nombreuses déclarations de satisfaction des divers pays européens). II relève toutefois que «le contrôle accru des frontières s'est accompagné de campagnes régulières de contrôle d'identité et d'interpellations dans les divers centres urbains ou dans les forêts avoisinant les présides occupés de Ceuta et Melilla, suivies d'opérations de refoulement vers la frontière algérienne ou mauritanienne. Ces campagnes ont donné lieu à de nombreuses violations des droits des migrants en situation irrégulière (interpellation de réfugiés, violences et mauvais traitements, refoulements sans saisine de la justice, etc.), qui s'ajoutent aux violences de délinquants et de trafiquants des êtres humains et aux violations subies par les migrant(e)s pendant leur long parcours migratoire, parfois avant même leur entrée sur le territoire national.

Les autorités arguent de leur droit de sanctionner l'entrée et le séjour illégaux, de combattre le trafic des êtres humains et de lutter contre les tentatives - tout aussi illégales - de franchissement des frontières internationales et notamment avec les Présides occupées (...).

75 Le Rapport préconise de « réviser et mettre à jour les lois relatives au droit d'asile, tout en veillant à leur harmonisation avec les normes internationales des droits de l'homme en la matière, de débattre de la manière de gérer les dispositions des conventions en matière d'asile, d'examiner et d'évaluer le rôle de la représentation du Haut-Commissariat des Nations Unies chargée des réfugiés au Maroc ».

$76 \mathrm{CNDH}$, "Conclusions et recommandations du rapport «Etrangers et droits de l'Homme au Maroc: pour une politique d'asile et d'immigration radicalement nouvelle $»$ : https://www.cndh.ma/fr/rapportsthematiques/ conclusions-et-recommandations-du-rapport-etrangers-et-droits-de-lhomme-au-maroc 
La dernière initiative fut la signature, en juin 2013, entre le Maroc, I'Union Européenne et 6 Etats membres d'une Déclaration conjointe établissant le partenariat de mobilité ».

Le Rapport conclut que les pouvoirs publics ne peuvent, dans l'accomplissement de ces missions, se soustraire aux dispositions constitutionnelles en matière de droits humains et de droits des étrangers, aux engagements internationaux contractés en vertu de la ratification de l'ensemble des instruments de protection des droits de l'Homme. II préconise en conséquence la nécessité d'adopter une nouvelle politique d'immigration et d'asile pour laquelle il formule une série de recommandations au gouvernement et à diverses parties prenantes. Elles réfèrent dans leur dimension normative à la convention de Genève et la nécessaire collaboration avec le HCR pour sa mise en œuvre, mais aussi aux conventions des droits humains pour le respect des droits des migrants. Elles distinguent à ce sujet entre les droits des migrants en situation régulière et ceux qui sont en situation irrégulière, sans faire mention explicitement à la Convention des Nations Unies sur la protection des travailleurs migrants et des membres de leur famille, quand bien même à la date où il a été présenté au Roi et a recueilli son approbation, le Haut-Commissariat chargé de cette convention procédait à l'examen du rapport initial du Maroc sur son application ${ }^{77}$.

Les événements tels que relatés par le Département des RME, désormais aussi en charge des migrants se sont succédés avec une rapidité extrême, aboutissant à la régularisation de nombreux réfugiés et migrants et à la formulation d'une stratégie nationale d'immigration et d'asile (SNIA):

- 9 septembre 2013, publication du rapport du CNDH;

- 10 septembre 2013, directives royales au gouvernement pour procéder à l'élaboration d'une nouvelle politique globale en matière d'immigration et d'un plan d'action opérationnel;

- 10 octobre 2013, création du ministère chargé de la migration;

- 6 novembre 2013, orientations royales pour « une nouvelle politique globale relative aux questions de l'immigration et s'asile suivant une approche humanitaire conforme aux engagements internationaux du Maroc et respectueuse des droits des immigrés »;

- 24 décembre 2013, distribution d'un premier lot de cartes aux réfugiés et migrants;

- 2 janvier 2014, lancement effectif des opérations de régularisation des étrangers en situation irrégulière;

- 18 décembre 2014, adoption en conseil du gouvernement de la SNIA.

77 N. Khrouz observe à ce sujet que le «hasard des agendas » a rendu le travail de la délégation officielle marocaine à Genève délicat. Cette délégation a en effet dû répondre à différentes questions concernant le respect des droits des étrangers au Maroc. De manière concomitante étaient diffusées les recommandations du CNDH appelant à une « politique d'asile et d'immigration radicalement nouvelle» et le premier communiqué royal soutenant une approche "globale et humaniste, conformément au droit international et dans le cadre d'une coopération multilatérale rénovée ». Le lendemain s'est tenue une réunion de travail, présidée par le Roi. Le communiqué du Cabinet royal qui la sanctionne souligne que «si la gestion opérationnelle de la question de l'immigration illégale connaît parfois certains excès, qui restent isolés, il n'y a pas d'usage systématique de violence de la part des forces de l'ordre et encore moins d'acharnement ». Partant, le Maroc refuse catégoriquement toutes les allégations fallacieuses qui tentent de porter atteinte à sa réputation: https://books.openedition.org/cjb/1129 
C'est par une simple circulaire conjointe du Ministère de l'intérieur et du nouveau Ministère chargé des Marocains résidant à l'étranger et des affaires de la migration, que l'opération exceptionnelle de régularisation de la situation de séjour des étrangers a été mise en place, sous l'autorité des walis et gouverneurs. Une première opération s'est déroulée durant toute l'année 2014. Après la mise en place d'une Commission nationale de recours en juin 2014, une seconde phase de régularisations a eu lieu tout le long de l'année. Près de 50000 migrants et réfugiés ont été régularisés. Les cartes d'immatriculation d'une durée d'une année délivrées aux immigrés les autorisent à travailler, et devraient leur faciliter l'accès aux services publics et aux prestations de l'OIM. Mais la question de la régularité de leur séjour risque de nouveau d'être posée à l'échéance, pour tous ceux qui n'auraient pas une activité génératrice de revenus suffisants.

La conception de la stratégie nationale d'immigration et d'asile (SNIA) s'est en fait affinée en marge de ces opérations. Son contenu apporte des solutions aux problèmes soulevés dans la discussion du rapport initial du Maroc, notamment en matière d'accès aux services publics ${ }^{78}$. Sa première évaluation quinquennale ${ }^{79}$ la décline sous mouture intégrée à travers 6 principes directeurs, 4 objectifs stratégiques, 11 programmes structurants, et 27 objectifs spécifiques pour lesquels 81 actions ont été prévues.

Leur examen ne renseigne pourtant pas sur le sort des migrants régularisés à l'échéance des titres de séjour d'une année qui leur ont été attribués, notamment s'ils n'accèdent pas à un travail permanent. II laisse aussi dans le doute le traitement à réserver aux migrants irréguliers qui demeurent exclus de la compétence du département ministériel.

La conduite de toutes ces opérations par voie d'instructions administratives exprime l'étendue des pouvoirs attribués aux instances sécuritaires et politiques, pour autant qu'elles disposent des moyens nécessaires à cet effet. Elle devrait être suivie de l'adoption d'une loi sur les réfugiés et l'asile, d'une autre sur les droits des immigrés, et de mesures diverses visant à éliminer du droit en vigueur toutes les restrictions aux droits des migrants prohibées par la Convention des Nations Unies précitée.

78 Voir Nations Unies CMW/C/MAR/1, Comité pour la protection des droits de tous les travailleurs migrants et des membres de leur famille, Examen des rapports présentés par les États parties en application de l'article 73 de la Convention, Rapport initial de l'État partie devant être soumis en 2004, Maroc, 12 juillet 2012; voir également O.N.U, Comité pour la protection de tous les travailleurs migrants et des membres de leur famille, XIXème session, Liste des points à traiter à l'occasion de l'examen du rapport initial du Maroc (CMW/C/MAR/1), Réponses du Maroc à la liste des points à traiter, 16 juillet 2013, CMW/C/MAR/Q/1/Add.1.

79 Ministère délégué chargé des Marocains résidant à l'étranger et des affaires de la migration, Politique nationale d'immigration et d'asile, 2018 : http://marocainsdumonde.gov.ma/wp-content/ uploads/2019/01/ Politique-Nationale-dimmigration-et-dAsile- -Rapport-2018.pdf 
Ce vaste chantier semble d'autant plus difficile à engager que la question de fond demeure celle de l'admissibilité d'une transhumance générée par un contexte géopolitique défavorable et la priorité donnée à la sécurisation des frontières nord ${ }^{80}$.

\section{Conclusion}

Le Maroc continue de se percevoir comme un pays d'émigration. Les conventions de main-d'œuvre qui lui ont historiquement conféré ce statut demeurent formellement en vigueur. Mais elles ne sont plus mises en œuvre depuis l'arrêt, au milieu des années 70, de l'émigration collective que les pays européens cosignataires organisaient et suscitaient à partir du Maroc. Les travailleurs marocains à l'étranger, dont une grande partie sont devenus citoyens des pays d'accueil, ne se déterminent plus par référence à leurs stipulations, mais plutôt par les directives européennes, les législations nationales et les conventions collectives qui imposent l'égalité de tous les travailleurs dans l'emploi et la protection sociale. La seule exception à cette évolution réside dans l'émigration temporaire des travailleuses agricoles vers l'Espagne, qui fait l'objet de procédures collectives encadrées de manière paritaire par les administrations des deux parties et les représentants des employeurs espagnols.

Dans la mesure où les conventions bilatérales de sécurité sociale régissent la portabilité des droits à la couverture sociale, elles étaient destinées à prendre effet au fil du temps grâce au retour définitif des travailleurs. Mais la transformation d'une émigration conçue comme étant limitée dans le temps en installation définitive dans le pays d'accueil exclut leur utilité pour les binationaux et les résidents permanent, et la réduit considérablement à l'égard des travailleurs irréguliers - peu souvent déclarés - qui remplissent rarement les conditions nécessaires pour prétendre aux prestations de la sécurité sociale. La désuétude de ces conventions fait place à des accords bilatéraux plus conformes à la stratégie européenne,

80 L'acquis le plus visible généré par la régularisation est d'ordre politique. Le Rapport du Département chargé des affaires de la migration précité rappelle que «la politique internationale du Maroc demeure particulièrement dynamique sur ce plan. Le Maroc a assuré la co-présidence avec l'Allemagne du Forum mondial pour la migration et le développement et la tenue à Marrakech de la 11 ème session de ce Forum, entre les 5 et 7 décembre 2018, ont permis de prendre la mesure de la haute estime dont elle jouit à travers le monde, au point qu'elle est reconnue parmi les bonnes pratiques les plus citées. Le leadership en la matière s'est affirmé au sein l'Union Africaine, lors du

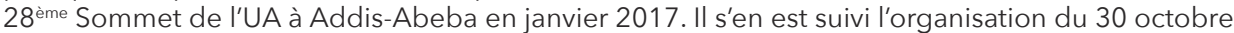
au $1^{\text {er }}$ novembre 2017, d'une Retraite régionale sur la migration, puis le 8 janvier suivant d'une Conférence ministérielle Africaine pour un Agenda africain sur la Migration à laquelle ont pris part une vingtaine de ministres et responsables gouvernementaux africains. Au 30 ème sommet de l'UA tenu le 29 janvier 2018 à Addis Abeba, le Maroc a présenté l'Agenda Africain pour la Migration qui renouvelle profondément le regard sur les migrations africaines et leur ouvre les perspectives d'une coopération Sud-Sud en tant que levier du co-développement et vecteur de solidarité. Le Sommet des Chefs d'Etat et de Gouvernement, à Nouakchott en juillet 2018, a d'ailleurs validé les mesures qu'il préconise et confirmé la proposition royale d'accueillir à Rabat le siège d'un Observatoire Africain pour la Migration.

Le Maroc a accueilli aussi le $1^{\text {er }}$ et le 2 mai 2018 à Marrakech la $5^{\text {ème }}$ Conférence ministérielle du dialogue Euro-Africain sur la migration et le développement. Outre la déclaration politique qui a sanctionné ses travaux, le Programme 2018-2020 qu'elle a adoptée est constitué d'un Plan d'Action qui traduit une approche régionale inclusive et pluripartite qui vise à renforcer les impacts positifs de la migration régulière, à mieux traiter les causes profondes des migrations irrégulières et à améliorer la collecte, l'analyse et le partage des données désagrégées y afférentes. 
visant l'attraction des profils rares et la collaboration des Etats tiers à la réduction de la migration par la coopération en matière de rapatriement et la maîtrise conjointe de la mobilité transfrontalière.

Cette coopération se déroule au niveau bilatéral entre les Etats de voisinage et dans le cadre euro-méditerranéen, depuis que I'Union Européenne a reçu mandat des Etats membres pour développer une politique commune en matière de migration. Son premier volet a pour objectif de développer, dans les sillages des Accords de Schengen, la libre circulation des citoyens européens et de restreindre l'accès des étrangers à l'emploi. Le second vise à sécuriser les frontières extérieures de l'Union contre l'arrivée des migrants et demandeurs d'asile, en mobilisant à cet effet l'assistance des organisations internationales et la coopération des Etats voisins. Ses manifestations juridiques et institutionnelles sont constituées au Maroc par l'adoption en 2003 de la Loi 02-03 sur la lutte contre la migration et l'immigration irrégulières et par le redéploiement des activités du HCR et de I'OIM dans le pays.

Les succès remportés par cette politique commune ont pour effet de gonfler le nombre des candidats marocains à l'émigration clandestine et de constituer, autour des points d'accès à l'Europe et de traversée de la Méditerranée, des foyers importants de Subsahariens décidés à poursuivre leur itinéraire migratoire au prix de leur vie. La dissuasion légale trouve vite ses limites à leur égard. Le recours aux mesures contestables d'arrestation, de déplacements et de reconduites collectives aux frontières, s'accompagnent forcément de l'irrespect des droits fondamentaux de ces migrants et d'abus de toutes sortes.

Du côté de l'UE, les ajustements apportés à cette politique ont pris la forme d'une approche globale de la mobilité, de la migration et du développement, fondée sur une responsabilité partagée et visant la collaboration active des pays d'origine, de destination et de transit. Du côté marocain, l'interpellation du Gouvernement à l'occasion de l'examen du rapport initial sur l'application de la Convention des Nations Unies relative à la protection des travailleurs migrants et des membres de leur famille a précipité l'annonce d'une nouvelle politique de l'immigration et de l'asile doublée de la régularisation de près de 50000 migrants et réfugiés, pour la plupart subsahariens.

Les deux démarches ont été accueillies favorablement par les pays d'origine. Elles doivent probablement leur succès politique davantage à leur concordance avec les orientations du consensus véhiculé par le Pacte de Marrakech autour de migrations sûres, ordonnées et régulières, qu'à leur aptitude à régler les problèmes posés par la migration irrégulière aux pays de voisinage de l'Europe.

Si pour les pays d'immigration qui se sont massivement abstenus de ratifier la convention précitée, les préconisations de ce Pacte ne constituent qu'une déclaration d'intention, en revanche, pour le Maroc et les autres pays qui y ont adhéré, elles actualisent et rappellent des obligations dont ils sont comptables à l'égard de tous les Etats dans le cadre des mécanismes de contrôle des Nations Unies.

Par cette nouvelle politique marocaine de migration, le pays s'engage objectivement à ouvrir le chantier de la mise en conformité des droits des immigrés avec les prescriptions de cet instrument international en éliminant les dispositions répressives de la Loi 02-03, abrogeant des règles législatives contraires et mettant en place de mesures protectrices des immigrés aussi bien en situation régulière qu'irrégulière. 
Alors que l'adhésion à la politique sécuritaire européenne transforme son territoire en espace de transhumance migratoire, les prescriptions de la Convention des Nations Unies l'identifient à la fois en tant qu'espace d'origine, de transit et d'emploi, tenu de garantir les droits qu'elles énoncent à tous les travailleurs migrants présents sur son territoire, au risque d'accroître son attractivité et la transhumance qui en découle.

C'est la voie que devrait emprunter le projet de loi sur l'immigration que promeuvent toutes les parties prenantes. C'est aussi celle qu'il soutiennent, pour l'adoption d'une loi sur les réfugiés et l'asile, sans revenir sur l'externalisation de la politique d'asile par l'Union Européenne, ni débattre de l'inadéquation de la Convention de Genève aux situations dans lesquelles le besoin de protection est imputable à la violence économique et aux effets du changement climatique, bien plus qu'à l'action des Etats et aux conséquences des guerres qu'on impose à leurs peuples.

\section{RACHID FILALI MEKNASSI}

Professeur, Université de Rabat.

\section{Thèmes de recherche: Droit social, droit du travail, droits de l'Homme.}

\section{Publications:}

$\sim$ R. Filali Meknassi et O. Tizaoui, « Regards à partir de l'expérience marocaine sur les limites d'une approche de la justice sociale fondée sur les droits au travail ", in I. Daugareilh (dir.), L'accès à la justice sociale - La place du juge et des corps intermédiaires, Ed. Bruyant, 2019, Bruxelles, p. 149.

R. Filali Meknassi, "Le droit à la sécurité sociale à l'ère de l'obsolescence programmée ", in I. Daugareilh et M. Badel (dir.), La sécurité sociale - Universalité et modernité, Ed. A. Pedone, 2019 , p. 243.

R. Filali Meknassi, "L'intégration des normes internationales du travail en droit marocain ", Revue de droit comparé du travail et de la sécurité sociale, 2017-1, p. 22. 